\title{
Numides, Numidie
}

\section{Mansour Ghaki, Jean-Pierre Laporte et Xavier Dupuis}

\section{OpenEdition}

Journals

Édition électronique

URL : https://journals.openedition.org/encyclopedieberbere/2768

DOI : 10.4000/encyclopedieberbere.2768

ISSN : 2262-7197

\section{Éditeur}

Peeters Publishers

\section{Édition imprimée}

Date de publication : 5 octobre 2012

Pagination : 5633-5668

ISBN : 978-90-429-2718-6

ISSN : $1015-7344$

\section{Référence électronique}

Mansour Ghaki, Jean-Pierre Laporte et Xavier Dupuis, « Numides, Numidie », Encyclopédie berbère [En ligne], 34 | 2012, document N76, mis en ligne le 15 décembre 2020, consulté le 17 février 2022. URL : http://journals.openedition.org/encyclopedieberbere/2768; DOI : https://doi.org/10.4000/ encyclopedieberbere. 2768

Ce document a été généré automatiquement le 17 février 2022.

(c) Tous droits réservés 


\section{Numides, Numidie}

\section{Mansour Ghaki, Jean-Pierre Laporte et Xavier Dupuis}

1 Avertissement : Les différentes composantes de cette notice ont été rédigées par X. Dupuis, M. Ghaki et J.P-Laporte. Les textes ont été revus et complétés par J. Desanges (Numidie ancienne) et N. Duval (Numidie tardive) qui ont ainsi contribué à l'effort commun.

\begin{tabular}{|l|l|}
\hline I. & Une géographie imprécise et variable \\
\hline II. & Une Numidie traditionnelle \\
\hline III. & La Numidie ancienne \\
\hline IV. & Les tribus de la Numidie \\
\hline V. & La Numidie romaine $($ de -46 à +429$)$ \\
\hline VI. & La Numidie chrétienne \\
\hline VII. & La Numidie vandale \\
\hline VIII. & La Numidie byzantine \\
\hline IX. & Chefs, rois et royaumes berbères de la fin de l'Antiquité \\
\hline X. & La Numidie et la conquête musulmane \\
\hline
\end{tabular}

\section{UNE GÉOGRAPHIE IMPRÉCISE ET VARIABLE}

2 On pourrait définir la Numidie comme le pays habité par les Numides, un ensemble de tribus manifestement mal connues des auteurs anciens, et dont aujourd'hui on serait bien en mal de donner une liste complète et/ou des localisations exactes. Des Numides sont attestés à l'époque romaine autour de Khamissa, antique Thubursicu, dite 
précisément Numidarum (« des Numides »), à Cellae, Masculula (Desanges 1962, p. 128), mais aussi par des bornes de la région de Bordj Bou Arreridj (ibid.., p. 66).

3 L'homophonie entre ce mot libyque et le grec Nomades a donné lieu à une interprétation grecque qui faisait des Numides des nomades. À la fin du I ${ }^{\text {er }}$ siècle avant J.-C., Strabon (II, 5, 33 ; XVII, 3, 15; cf. Luisi 1979) s'en fait l'écho. On sait aujourd'hui qu'elle est inexacte. La région comportait des villes bien avant la période romaine, et sans doute un éventail de types de vie nomade, semi-nomade et sédentaire beaucoup plus varié qu'on ne le croit d'habitude.

4 Au même titre que " maure* ", « afer » et « libyen* ", le terme " numide » pourrait être à l'origine un ethnonyme porté par une ou des tribus déterminées, qui aurait été étendu à d'immenses territoires par des étrangers, notamment des géographes grecs et romains, qui ne disposaient que de fort peu d'informations, et hésitaient d'ailleurs euxmêmes. C'est ainsi que Polybe (III, 33, 15) et Tite-Live (XXIV, 49, 5) considéraient les Maures (Maurusii) comme une catégorie de Numides (Desanges 1999, p. 183), tandis qu'au II siècle de notre ère, Hérodien (VII, 9,1) voyait dans les Numides une fraction des Maurusii (Desanges 1999, p.196). Numide et Numidie paraissent des noms «donnés » par d'autres, comme « libyen » ou plus tard "africain» (M. Ghaki 1983 et 2005). Il est loin d'être sûr que les autochtones qui n'appartenaient pas aux tribus éponymes se reconnaissaient dans ces vocables. Le terme " Numides » recula au fil du temps devant le terme "Maures", qui finit par recouvrir aux yeux des Byzantins tout le monde tribal, souvent insoumis.

5 Dès que l'on examine les données en détail, la situation se complique. Les termes « Numide » et « Numidie » désignaient en fait des ensembles et des régions qui d'une part ne se recouvraient que partiellement à une même époque et qui d'autre part variaient selon les périodes (parfois même pour chaque sujet) selon les critères employés: géographiques, ethniques, culturels, religieux, politiques, militaires, administratifs, etc. (Desanges 1980 et 1999 ; pour les limites politiques, voir de manière pratique M. Benabbès 2011, notamment les cartes p. 288-293). Cela impose d'être explicite quant aux critères retenus pour chaque période : temps antérieurs aux rois, royaumes, dominations romaine, vandale et byzantine. L'histoire des régions numides est à la fois traversée et sous-tendue par des réalités diachroniques comme les tribus, le christianisme et les rois tardifs qui méritent un examen particulier.

Cependant, il semble avoir longtemps existé en quelque sorte en arrière-plan des avatars politiques et militaires de l'Afrique du Nord antique, une Numidie que l'on pourrait qualifier de traditionnelle, dans la mesure où cette notion persista à travers les âges dans l'esprit des Anciens.

\section{UNE NUMIDIE « TRADITIONNELLE »}

7 La Numidie «traditionnelle» pourrait être en gros définie (Desanges 1980 et 1999) comme un espace limité au nord par la Méditerranée, à l'ouest par l'Oued el-Kebir (antique Ampsaga) et à l'est par la Fossa regia, qui sépara le territoire du royaume massyle* d'abord de celui de Carthage puis de celui de Rome immédiatement après 146. La limite méridionale est plus floue, selon qu'on lui annexe ou non des territoires gétules* dans lesquels les autorités de toutes les époques (y compris royale) eurent des 
difficultés à s'imposer, mais qui appartinrent sans aucun doute à la Numidie chrétienne.

8 Cette région «traditionnelle " présente des caractères physiques et géographiques divers, du nord au sud, mais aussi, de manière moins tranchée d'est en ouest.

9 La partie nord est bordée partiellement par des chaînes de montagne peu élevées qui bénéficient de précipitations assez abondantes. Viennent ensuite les Hautes plaines jusqu'au pied de l'Aurès. Elles représentent le pays des céréales, objet déjà d'exportation au temps de Massinissa*, mais aussi des chevaux, "production" importante de la Numidie (Strabon, 17, 3, 7 ; cf. Gsell, HAAN, V, p. 181). À l'époque romaine, leur élevage est attesté par les mosaïques de l'oued Athmenia, au sud-ouest de Cirta, mais aussi par les haras de l'hadrumétin Sorothus, à Aïn Babouche, près d'Oum elBouaghi (Laporte 2006, p. 1373-1375). Plus au sud, se dressent le massif de l'Aurès, peuplé de sédentaires, et au sud-est les monts des Nemencha*, ces derniers présentant des caractères steppiques et occupés plutôt par des semi-nomades et/ou des transhumants, et même par des sédentaires dans les oasis de la frange méridionale.

Ces différences de climat et de précipitations se traduisaient dans les modalités de l'occupation humaine : au nord, des Numides agriculteurs et sédentaires; au sud, des Gétules, sans doute en partie nomades (mais pas tous), sur des territoires dont l'appartenance au royaume massyle pose question, leurs habitants s'opposant aux rois. Mais cette opposition n'était pas permanente, dans la mesure où le Médracen*, mausolée d'un roi inconnu, de la fin du $\mathrm{IV}^{\mathrm{e}}$ ou du début du $\mathrm{III}^{\mathrm{e}}$ siècle, se situait au contact de l'Aurès. Dans le même sens, le fait que Tébessa*, ville incontestablement numide, se trouvait au sud du territoire principal des Musulames*, peuple que l'on sait maintenant gétule (Laporte et M'charek 2010) montre que la dimension ethnique des mots « gétule » et «numide » ne coïncidait pas avec leur contenu géographique dans la théorie des « climats » chère aux géographes antiques.

\section{LA NUMIDIE ANCIENNE}

\section{La Numidie avant les rois}

$11 \mathrm{Au} \mathrm{V}$ siècle avant J.-C., Hérodote (Histoire II, 32) ne parle que des Libyens, divisés en de nombreuses peuplades, dont des sédentaires et des nomades. Par la suite, les géographes antiques parlèrent d'une immense Numidie, anciennement divisée en deux entités (masaesyle à l'ouest et massyle à l'est), auxquelles il faut sans doute ajouter une partie méridionale difficile à délimiter, souvent semi-nomade, et donc aussi semisédentaire (voir une présentation générale dans Bouchenaki 1979).

Pour Strabon (XVII, 3,9) à la fin du I ${ }^{\text {er }}$ siècle, le territoire des Maurusii* (Maures) faisait suite à l'ouest à celui des Masaesyles*, qui commençait pour sa part au fleuve Molochath (Moulouya*) et se terminait au cap Tréton (Bougaroun).

13 Aux époques protohistoriques, avec les difficultés que comporte ici la notion de Protohistoire, toute l'Afrique du Nord participait de la même culture libyque, partageant une langue, des croyances, des rites et coutumes funéraires, une écriture, etc.

14 Cette culture comportait à la fois une certaine unité et des différences régionales marquées (Camps 1999 et 2000). Des cartes de répartition permettent d'expliquer, dans 
une certaine mesure, des limites que l'on retrouve bien plus tard (Camps 1999). La limite sud de la culture des céréales représente par exemple plus ou moins celle de la Numidie "sédentaire " et donc aussi la limite nord de la Numidie "semi-nomade " (mais l'Aurès échappe à cette particularité).

Ici comme souvent ailleurs, les sépultures sont les éléments de civilisation les mieux connus. Les modes de sépulture montrent en fait une grande diversité : tumuli, dolmens*, haouanet*, bazinas*, etc., différents suivant les régions, à la fois par une plus ou moins grande densité, mais surtout, un plus ou moins grand pourcentage de chaque type.

Dans le domaine funéraire, on note par exemple au nord-ouest de nombreux haouanet, également attestés de manière sporadique jusque dans la région de Tébessa, ainsi à Gastel*. Le centre-ouest tunisien montre de nombreux dolmens. Le sud comporte majoritairement des bazinas, avec des tumuli devenant plus nombreux à mesure que l'on se rapproche du Sahara. Dans la région de Négrine*, on note même de curieux tumuli « à chapelle tréflée ", de tradition apparemment saharienne.

17 Cette diversité géographique se retrouve aussi dans le temps. D'aucuns mettent encore l'accent sur la " permanence ", la "résistance au changement », la "fixité », etc, les changements venant exclusivement de l'extérieur. On insistera beaucoup plus maintenant sur les évolutions internes. Le dépôt de mobilier funéraire, anciennement rare, sinon inexistant, apparut et devint la règle. La sépulture individuelle se répandit ; la continuité de la céramique modelée se fit parallèlement à l'introduction de la poterie tournée, des épitaphes, de la pierre de taille, etc. L'architecture funéraire mégalithique, qui ne venait pas d'ailleurs, évolua. Ainsi apparurent à l'est des structures mégalithiques complexes : Ellès, Makthar*, Zouakra, etc. On peut également mettre en perspective les grands mégalithes de Makthar, et des monuments d'origine tout aussi autochtone comme le Medracen*, le Tombeau de la chrétienne*, les Djedars*, etc.

Qu'il s'agisse de tumuli, de dolmens, bazinas, ou autres, plusieurs situations se présentent :

- des tombes isolées, dispersées, une tombe ou deux éloignées du reste, notamment dans le "pays des tumuli",

- des nécropoles regroupées, grandes ou petites, qui se divisent elles-mêmes en deux types: soit à l'écart de tout habitat, soit à proximité de centres urbains, avec des chronologies apparemment différentes. À Bulla Regia*, une nécropole dolménique avoisine une cité numido-romaine. À Dougga*, une nécropole dolménique est proche de la cité antique. À Makthar, une nécropole mégalithique (dont certains des grands monuments ont encore été utilisés au III siècle après J.-C.!) avoisine la cité numide punicisée, puis romanisée (ce fut d'ailleurs le même espace funéraire durant toute l'antiquité, et le cimetière musulman le flanque encore).

19 Les nécropoles semblent être devenues des points de ralliement, de rencontres périodiques, en générant avec le temps un habitat fixe plus ou moins important. Une carte de répartition des inscriptions libyques (Camps, 1999, p. 58, fig. 2 ; ici fig. 1) les montre situées pour l'essentiel dans les régions de sédentaires, celles des «villes numides », des grandes nécropoles, où la densité de population était sans doute la plus forte. 


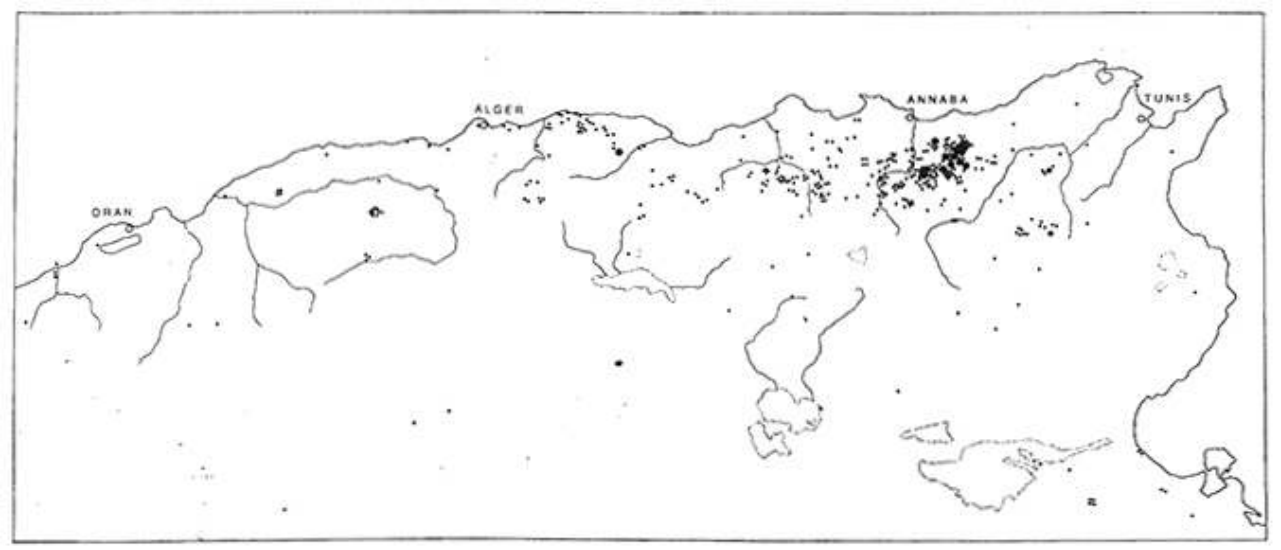

Fig. 1. CARTE de RÉPARTition des INSCRIPTIONS LIBYQUes (CAMPS 1999, P. 58, fig. 2)

L'archéologie témoigne de l'ancienneté de certaines agglomérations qui remontent à des périodes pour lesquelles les sources historiques manquent. Une tombe de Mididi est datée du $\mathrm{VI}^{\mathrm{e}}$ siècle avant notre ère. De grandes bazinas découvertes dans les années 1990 sous une place d'époque romaine de Chemtou semblent bien témoigner de l'existence d'un centre urbain préromain, etc. On peut maintenant admettre que des agglomérations (habitat fixe, concentré, permanent) ont existé dès le VI ${ }^{e}$ siècle avant J.C., même si dans l'ensemble on les connaît mal. Plusieurs villes, comme Dougga, sont qualifiées de «polis » lors de l'invasion d'Agathocle (310 avant J.-C.) lorsque le tyran de Syracuse va y chercher du butin pour ses troupes. Dans ce cas, on parlera de « cité d'une certaine grandeur " vers 310 et d'une "polis» au second siècle avant J.-C. grâce aux inscriptions en libyque horizontal qui mentionnent une organisation municipale. D'autres agglomérations sont citées lors de la guerre de Jugurtha. Toutes ces villes étaient bien numides, car Carthage ne semble avoir fondé aucune cité à l'intérieur du pays.

Dès le milieu du premier millénaire avant J.-C., la culture libyque, déjà diversifiée, était plus ou moins marquée suivant les régions par des influences puniques, à partir non pas de la seule Carthage, mais aussi des cités libyco-puniques installées sur le littoral (Lipiński 1992, Krandel-Ben Younès 2002). Les « faciès » archéologiques particuliers que l'on rencontre au Sahel, à Mogador, à Rachgoun (dès le VII siècle avant notre ère), etc., en sont la preuve. Dans la conception mise en valeur par P. Cintas vers 1950, toutes les cités côtières auraient été des fondations puniques, réparties tous les 30 ou $40 \mathrm{~km}$, distance maximale d'une navigation* uniquement diurne, et fondées suivant un " programme » d'ensemble. Un demi-siècle plus tard, le schéma est différent (Laporte 2010). D'une part on a fortement réévalué les capacités nautiques des bateaux puniques, et même phéniciens, capables de naviguer de nuit; d'autre part les distances sont moins régulières qu'on ne l'a dit. Les rares points que l'on pourrait dire "phéniciens » sont très éloignés. Les sites qualifiés de "puniques", en fait libyco-puniques, plus rapprochés, mais d'importance très inégale, datent pour la plupart de la fin de la punicisation, et sont souvent attestés pour des périodes pendant lesquelles ils dépendaient des rois numides. Par ailleurs, certaines régions comme le nord de la Tunisie (Khroumirie*), la Kabylie*, le Rif* étaient peu favorables, voire hostiles, et on voit mal comment Carthage aurait pu y maintenir de force des enclaves ressenties comme étrangères. La punicisation de l'intérieur semble avoir progressé à partir de certaines villes côtières, dites puniques, mais dont nous connaissons en fait mal le 
statut, probablement plus indépendant de Carthage qu'on ne l'a dit, si ce n'est pendant des crises (par exemple lorsqu'Hannibal leva des soldats dans les villes du Métagonium, mais on était alors à la période royale).

\section{La Numidie royale}

\section{L'Ancienneté des royaumes} masaesyle comme des acteurs actifs et importants dans les relations entre Rome et Carthage, comme partenaires de l'un et donc adversaires de l'autre, et l'on voit se dessiner les deux royaumes considérés comme numides un peu avant le déclenchement de la guerre. Même dans ce cas, le vocabulaire était imprécis. Le roi des Masaesyles, Syphax, dominait l'ouest algérien à partir de sa capitale, Siga, mais il apparaissait aussi comme une sorte de suzerain dans la région du détroit de Gibraltar (Desanges 1999, p. 183, n. 17).

L'existence de royaume(s) est sans doute fort ancienne, mais les plus anciens rois numides susceptibles d'avoir régné sur de larges territoires sont restés inconnus des sources gréco-romaines classiques. L'un d'eux se manifesta au début du $\mathrm{III}^{\mathrm{e}}$ siècle, voire à la fin du IV e siècle avant notre ère, avec la construction du Médracen*. Il était assez puissant pour faire construire ce gigantesque monument, avec une remarquable capacité à mobiliser une main d'œuvre abondante autour d'un projet élaboré, profondément libyque (une gigantesque bazina), avec des références architecturales méditerranéennes (gréco-puniques) précises dans la colonnade engagée du pourtour.

La Numidie n'apparut réellement dans l'Histoire que lors de la première guerre punique (264-241), lorsque les historiens grecs et romains commencèrent à s'intéresser aux affaires africaines (pour autant qu'elles concernaient Rome). Pour eux, la notion de Numidie recouvrait alors l'ensemble de l'Afrique du Nord à l'exception de la Maurétanie (en gros le nord de l'actuel Maroc) à l'ouest et, à l'est, du territoire carthaginois (l'angle nord-est de l'actuelle Tunisie).

Le seul exposé d'ensemble sur les rois et royaumes numides reste contenu dans les tomes 5, 6 et 7 de l'Histoire ancienne de l'Afrique du Nord de S. Gsell, même si nombre de points ont été précisés depuis, notamment par G. Camps en 1960.

Ce n'est qu'à partir de Gaia* en pays massyle et de Syphax* en pays masaesyle que l'on commence à appréhender quelques détails, et que la liste des rois numides devient presque complète jusqu'à la fin du royaume (46 avant J.-C.). On se reportera de manière pratique au tableau généalogique donné par $\mathrm{M}$. Coltelloni-Trannoy dans l'article Juba* (EB, XXXV, 2003, col 3914-3938).

À l'est, Gaia régnait sur les Massyles, dont le territoire s'étendait jusqu'aux territoires carthaginois, avec des limites contestées (puisque plus tard son fils Massinissa put revendiquer des territoires que lui avait enlevés (arthage).

Faute d'intérêt direct des historiens grecs et romains (à l'exception, limitée, de Polybe) pour ce qui ne concernait pas les relations entre Rome et Carthage, on connaît mal le fonctionnement interne des royaumes numides, qui possédaient une organisation propre (Ghaki 1993). Ils ne revêtaient pas la forme de monarchies absolues, mais devaient s'appuyer d'une part sur des villes relativement autonomes, et d'autre part sur des allégeances tribales parfois fragiles. C'est ainsi que l'on vit des régions entières 
passer d'un roi à un autre, ainsi de Massinissa à Syphax, puis inversement, après une bataille. Les rapports entre les rois et les villes numides commencent à être mieux connus (Ghaki, sous presse); leurs rapports avec les tribus (autre que leur tribu d'origine) restent à documenter.

\section{Le royaume numide sous Massinissa et Micipsa} (118 avant J.-C.) au moins. maximale. relatives à chaque souverain.

La mort de Gaia, suivie d'une crise dynastique, entraîna le retour en Afrique de Massinissa qui récupéra son royaume par les armes. Mais quelque temps plus tard, Syphax, roi des Masaesyles, s'empara du royaume massyle. Dans les années qui suivirent Zama* (202 avant J.-C), les deux royaumes furent réunis par Massinissa, qui forma ce que l'on pourrait appeler une " grande Numidie».

Dans la première moitié $d u \mathrm{II}^{\mathrm{e}}$ siècle, le royaume numide s'étendait entre les Fosses phéniciennes qui séparaient le royaume de Massinissa du territoire de Carthage à l'est, et à l'ouest la Mulucha (Moulouya), borne occidentale du royaume conquis sur Syphax.

La paix acceptée par Carthage en 201 lui interdisait de déclarer la guerre à qui que ce soit sans l'aval de Rome, ce qui laissait en fait les mains libres à Massinissa. Profitant de toutes les occasions, lorsque Carthage était affaiblie et/ou Rome occupée ailleurs, le roi enleva à plusieurs reprises, mal datées (Desanges 1995), des territoires pris par Carthage à ses ancêtres, notamment la Tusca, dont on distingue bien, aujourd'hui, les contours et l'importance économique et politique (M'Charek, 1999). La frontière orientale avec le territoire punique se déplaça vers l'est jusqu'à la future Fossa regia. Puis Massinissa s'empara des Emporia que jamais ses prédécesseurs n'avaient dominés. On peut alors parler non pas de Numidie mais de royaume numide; celui-ci atteignit alors son extension maximale depuis la Moulouya à l'ouest jusqu'aux Autels des Philènes à l'est. Son étendue semble être restée identique jusqu'à la mort de Micipsa*

\section{La Numidie après la mort de Micipsa (118 avant J.-C.)}

Après la destruction de Carthage en 146 avant J.-C., Rome annexa sous l'appellation de provincia Africa tout le territoire que Carthage occupait encore, à l'intérieur de la future Fossa regia, une ligne sinueuse qui partait, au nord, de l'embouchure du fleuve Tusca (Oued el-Kebir, près de Tabarka) pour aboutir, sur la côte orientale de la Tunisie, à Thaenoe (Henchir Tina, au sud de Sfax). La Numidie royale garda son extension

Après la mort de Micipsa (118 avant J.-C.) le royaume numide fut d'abord partagé en trois royaumes distincts entre Adherbal*, Hiempsal* et Jugurtha*, avant que ce dernier ne les réunît entre ses seules mains par le meurtre et la guerre.

Puis l'étendue des royaumes numides se restreignit à plusieurs reprises, avec l'intensification et l'élargissement de la présence romaine à l'est, et l'avance des royaumes maures à l'ouest. On trouvera les détails de cette diminution dans les notices

Les modifications de territoire les plus importantes datent de Jugurtha qui tenta de reconstituer le royaume de son ancêtre Massinissa, mais se heurta à Rome (111-105), dont il vainquit les armées à plusieurs reprises. Une difficulté naît de l'imprécision géographique des récits de Salluste, sur une région qu'il connaissait pourtant bien. Dès 
les années 1950, jusqu'à une synthèse parue en 1981, partant d'une localisation ancienne exacte, à Kalaat Senane, du "fortin de la Mulucha » pris par Marius, André Berthier a proposé une série d'identifications très différentes de celles que l'on admet d'ordinaire, en plaçant notamment Cirta, non plus à Constantine, mais au Kef. Cette identification n'a dans l'ensemble pas été suivie par le monde savant, tout en conservant par ailleurs de chauds partisans, que ne sauraient convaincre des réfutations pourtant en règle (ainsi celles de Desanges 1982, 1974, 1987, et à paraître). Vers la fin de son règne, Jugurtha donna à son beau-père Bocchus Ier*, roi des Maures, le tiers occidental de son royaume pour prix de son alliance contre les Romains. Bocchus s'en fit confirmer la possession par Rome en livrant son gendre, qui fut emmené à Rome puis exécuté. Après la fin de Jugurtha, Lepcis Magna et la région des Emporia semblent avoir fait sécession du royaume numide dont les séparaient d'une part leur vieille tradition urbaine punique et d'autre part de vastes solitudes parcourues par des Gétules errants (Desanges 1999, p. 185). Le royaume numide ainsi amputé tendit à se rapprocher de ce que nous considérons comme la Numidie traditionnelle.

C'est avec Juba I ${ }^{\text {er }}$ (85-46 avant J.-C.) que s'acheva la royauté numide. À côté de la majorité de son monnayage légendé en punique, il fit frapper au moins une monnaie avec la légende d'avers Rex Iuba, en latin, alors que le revers restait en punique (Alexandropoulos 2000, p. 401, n²9). En prenant le parti de Pompée, il fit le mauvais choix. Après la victoire de Thapsus (46 avant J.-C.), César réorganisa l'Afrique. La Numidie royale disparut. Elle fut amputée de sa partie occidentale annexée par le roi maure Bocchus le Jeune (49-33). La frontière romaine avec la Maurétanie fut fixée à l'Ampsaga (oued el-Kébir du Constantinois). C'est peut-être à cette occasion que l'Aurès fut donné à la Maurétanie, car on le retrouve plus tard en possession de Juba II (Desanges 1964; Laporte 2003a, p.190-191). Ce qui restait de la Numidie royale orientale fut annexé par Rome et démembré comme on le verra plus loin dans la partie consacrée à la période romaine.

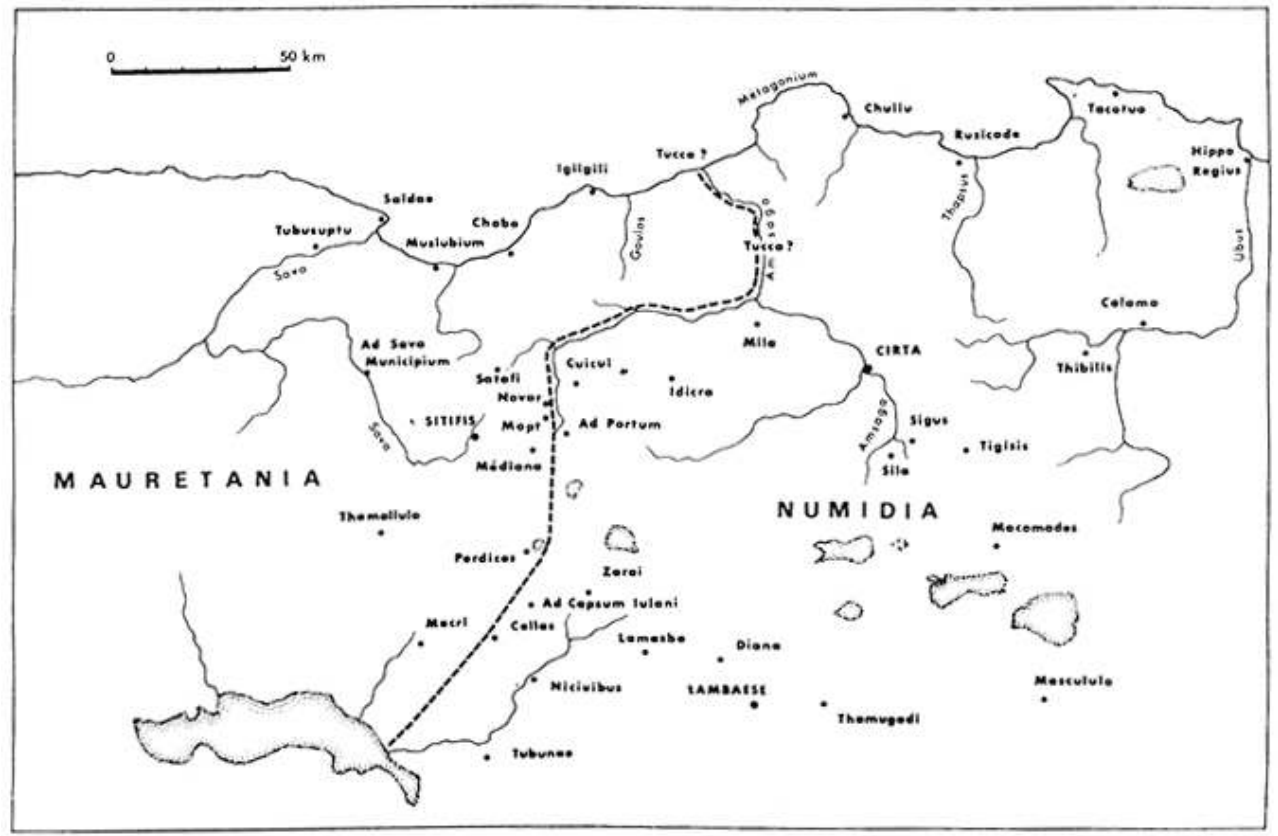

Fig. 2. CARTE DE LA FRONTIÈre TRADITIONNELLE ENTRE MAURÉTANIE ET NUMIDIE. D'APRÈS CAMPS 1999, P. 67, fig. 11. 


\section{Urbanisation et culture à l'époque royale} une organisation qui en fit peu à peu des villes telles que nous les entendons. Bien qu'elles aient gardé une organisation interne où subsistaient des titres et des fonctions libyques intraduisibles en punique (Chaker 1986, Ghaki 1993), certaines d'entre elles semblent avoir été fortement marquées par le modèle punique (Camps 1979). Ceci tint certainement au rayonnement, non seulement économique, mais aussi culturel, de Carthage, où avaient été éduqués nombre de jeunes princes libyques, dont un certain nombre épousèrent des princesses puniques (Sophonisbe* n'en est que l'exemple le plus connu). Plusieurs facteurs renforcèrent cette tendance. C'est ainsi que le règne de Massinissa vit la reconquête d'anciennes cités numides punicisées pendant l'occupation carthaginoise qui avait suivi leur perte par Gaia, souvent dirigées plus tard par des suffètes. D'autres villes purement numides, mais pas toutes, semblent avoir adopté des règles semblables. Le phénomène pourrait d'ailleurs avoir été lié à la mise en place du pouvoir royal.

L'archéologie révèle des villes anonymes souvent fortifiées (ce qui montre qu'il y avait des conflits), l'histoire nomme de vieilles cités qui avaient hébergé le trésor royal (Thala, Capsa, etc.), la toponymie atteste de villes qualifiées de Regius ou Regia à l'époque romaine (Bulla, Hippo, etc).

Des régions déterminées virent une implantation de populations punico-phones suffisamment denses pour que leur langue se maintienne au sud d'Hippone jusqu'à l'époque de saint Augustin voire au-delà (Camps 1994, Lepelley 2005). L'accueil probable de réfugiés puniques après la chute de Carthage pourrait expliquer en partie leur implantation primitive dont on ignore toutefois la date et les circonstances.

Le punique, langue internationale de l'époque, était aussi celle du pouvoir numide. Les monnaies royales numides, apparues à la fin $\mathrm{du}_{\mathrm{III}}^{\mathrm{e}}$ siècle, portèrent des légendes puniques jusqu'au $\mathrm{I}^{\mathrm{er}}$ siècle avant J.-C. (Alexandropoulos 2000). portraits. Mais rapidement Syphax, puis Massinissa, furent représentés sous une forme idéale hellénisée, symbole d'une nouvelle influence culturelle. partiellement hellénisées à partir du règne de Massinissa (Camps 1995, Desanges 2007). Trois facteurs facilitèrent cette hellénisation :

- les influences reçues de Carthage (à cette époque, l'art punique était déjà notablement hellénisé lui-même),

- les rapports directs des Numides avec le monde grec, notamment à l'initiative de Massinissa, et pendant tout le règne de Micipsa,

- enfin les idées et les formes gréco-romaines introduites dans le cadre des rapports avec Rome pour laquelle la civilisation grecque représentait une valeur culturelle supérieure à la sienne propre.

Le résultat fut un "hellénisme numide » indéniable dans les villes royales et dans le comportement des élites (Desanges 1989, Camps 1995) : représentations des rois sur leurs monnaies (dont les légendes en alphabet néopunique furent parfois remplacées par des légendes en caractères grecs, cf. Alexandropoulos 2002), introduction de la symbolique hellénistique dans les stèles votives consacrées à Baal dans les sanctuaires 
numido-puniques, etc. On peut certes s'interroger sur la diffusion de cet hellénisme audelà des villes royales, mais des traits helléniques ou hellénistiques apparaissent de manière sporadique dans les décors des mausolées-tours. On en trouve également la trace sur des peintures dans des haouanet, ainsi celles de Sidi M'hamed Latrech (Ghaki 1999).

Les régions numides présentent de grands mausolées* que l'on attribue à des rois. Les datations reposent sur des critères stylistiques flous, et interfèrent souvent avec l'attribution, non prouvée, à un personnage déterminé. Plusieurs sont placés à des dates variables du IIe s. avant J.-C., ainsi celui du Khroub. De son cote, le Médracen* remonte à une période beaucoup plus ancienne : fin IV ${ }^{\mathrm{e}}$-début III ${ }^{\mathrm{e}}$ siècle (Camps 1973 et 2010). On confond souvent "architecture royale» (monument destiné à un roi, un prince, etc.) et " architecture d'époque royale » (un notable pouvait très bien se faire construire un grand mausolée). Si l'on peut accepter l'attribution du Médracen a un roi (encore indéterminé), il ne peut en être de même des deux mausolées A et B de Sabratha*, ville où il n'y eut jamais de rois. Les différents raisonnements présentés demandent à être revus, dans la mesure où l'on connaît plus de monuments "royaux " que de rois attestés et où ils ne se trouvent pas toujours là où on les attendrait. Le cas du mausolée de Dougga est très parlant : on a confondu les deux textes bilingues RIL 1 et 2 pour parler du mausolée de Massinissa, puis on a réalisé que Massinissa était plus probablement enseveli dans la région de sa capitale principale, Cirta. On a parlé alors de cénotaphe, bien que cette pratique soit inconnue chez les Numides; on a été jusqu'a « corriger » la bilingue du mausolée (RIL 1) pour faire d'Atban, un architecte, etc. Une révision de ces mausolées a été entamée (Camps 1973 et 2010, Laporte 2008, Kherbouche et Laporte 2010), mais elle devra être poursuivie.

\section{LES TRIBUS DANS L'HISTOIRE DE L'AFRIQUE DU NORD ET DE LA NUMIDIE}

Sous le vocable très imprécis de "tribus ", terme hélas assez dépréciatif en français, on englobe des structures autochtones de niveaux relatifs variés (et souvent inconnus): tribu, confédération, etc. Nous l'emploierons ici dans le sens de "peuple», et de mode d'organisation différent de celui des cités puniques ou organisées « a la punique » (puis "à la romaine»). L'ouvrage fondamental en ce qui concerne les tribus antiques d'Afrique du Nord reste le Catalogue de J. Desanges, paru en 1962 avec un précieux classement géographique. Le même auteur en donne maintenant une mise à jour en traitant de chacune à sa place alphabétique dans l'Encyclopédie berbère.

Les tribus sont le fondement même de l'histoire de l'Afrique du Nord antique, médiévale et même souvent moderne. Par un effet de source, elles sont quasi inconnues pendant la Protohistoire, n'apparaissent que de manière sporadique pendant la période royale, pour transparaître de manière plus forte pendant les périodes romaine, vandale et byzantine, et arriver enfin en pleine lumière pendant le Moyen Age, comme l'illustre parfaitement l'Histoire des Berbères d'Ibn Khaldoun.

Bien plus anciennes que le pouvoir royal, puisque l'expansion de certaines d'entre elles en était la source, les tribus sont attestées dès l'époque royale, si l'on rapporte à cette période la plupart des inscriptions libyques. Elles ne sont mentionnées que plus tard par les historiens grecs et latins, dans des textes qui se rapportent tout d'abord aux 
affrontements du début de notre ère (Rachet 1970, Bénabou 1976), période pendant laquelle l'armée romaine étendit son emprise en progressant pour l'essentiel le long d'une très ancienne route de Carthage à Theveste, qui allait devenir à l'époque romaine la «route de Numidie ». Cette avancée vers le sud et le sud-ouest à partir de l'Africa vetus fut lente devant la résistance opposée par les populations gétules semi-nomades et nomades, notamment celle des Musulames* sous la conduite de Tacfarinas*.

Les indices liés au récit des affrontements guerriers rapportés par les historiens anciens, mais aussi à la lecture et à la répartition des inscriptions libyques, puniques et latines permettent peu à peu de dessiner les territoires de plusieurs de ces tribus et/ou confédérations pendant l'Antiquité.

Des progrès méthodologiques considérables dans la délimitation des territoires ont été apportés de manière exemplaire en ce qui concerne par exemple les Misiciri* (Camps 1993) situés au milieu de la Numidie traditionnelle. Plus récemment, A. M’charek (1999 et 2001) a enrichi et synthétisé les résultats de travaux de prospection anciens et récents en précisant le territoire des Musulames. Il s'agissait pour l'essentiel de tribus d'éleveurs nomades ou semi-nomades qui avaient besoin de très grands espaces pour leurs troupeaux, sans ignorer ni les cultures en fond de vallée, ni les villes.

51 La sensible amélioration récente des connaissances amènera à appréhender de manière plus précise un phénomène jadis négligé, voire ignoré : le rôle de certaines tribus dans la transformation de leur chef-lieu en ville romaine (alors qu'on y voyait jadis simplement une fondation romaine). C'est ainsi que dans l'appellation "romaine » de Thubursicu Numidarum (Khamissa), Numidarum n'est pas une simple indication géographique, mais bien une précision d'origine politico-ethnique : la structuration «à la romaine » de son territoire par l'une des tribus qui se disaient elles-mêmes Numides.

Depuis les indépendances des pays du Maghreb, les études concernant les tribus médiévales ont pris une ampleur nouvelle, et, avec elles, on accède à une meilleure compréhension de l'histoire de l'Afrique du Nord sur la longue durée. On peut maintenant discerner pour un nombre croissant de tribus à la fois des continuités et des changements dans la durée. La composition et donc le nom des confédérations ont varié dans le temps. Depuis l'Antiquité, les tribus libyco-berbères ont cédé de la place à (ou ont été absorbées par) des tribus arabes venues d'Orient. Leurs localisations ont parfois fortement varié depuis le Haut Moyen Age. Certaines tribus ont disparu, comme les Kotamas*, attestés dans la Kabylie des Babors, dès le second siècle après J.-C., à l'époque byzantine et encore au début de la période musulmane, jusqu'à ce qu'ils se consument au service des Fatimides*. En revanche, on a noté récemment des exemples étonnants de permanence, comme en Tunisie celui des Gunzuzi (M'charek, à paraitre), qui ont gardé à la fois le même nom et le même territoire de l'Antiquité jusqu'à nos jours.

53 Les recherches et découvertes récentes doivent permettre de reprendre maintenant sur des bases solides et d'étendre à des régions plus vastes la grande fresque historique (de l'Antiquité à nos jours) qu'avait dressée en 1913 le contrôleur civil et géographe Ch. Monchicourt (1913, p. 251-269 et suivantes) sur les tribus de la région située entre M'daourouch (Madauros), Henchir Guergour (Masculula), Dougga (Thugga), Téboursouk (Thubursicu Bure), La Kessera (Chusira), Kasserine (Cillium) et Tébessa (Theveste), c'est-àdire en plein cœur de l'ancienne Numidie. 


\section{LA NUMIDIE ROMAINE (de 46 avant J.-C. à 429 après J.- C.)}

Les événements liés aux guerres civiles de la fin de la République provoquèrent la disparition du royaume numide de Juba Ier, dont la partie orientale constitua dès 46 avant J.-C., suite à la victoire de César à Thapsus, la province d'Africa Noua, tandis que l'actuel Constantinois, limité à l'ouest par l'oued El-Kebir (l'antique Ampsaga), passait sous l'autorité d'un aventurier campanien, P. Sittius, qui avait eu l'intelligence de prendre le parti de César. Après l'assassinat de ce dernier, Sittius lui-même disparut rapidement, et son « état ", d'abord repris par Arabion*, fils du roi Mastenissa, fut très vite rattaché à l'Africa Noua. L'ensemble de l'Afrique, réunifiée, passa ensuite sous l'autorité de Lépide puis d'Octave (Desanges 1993). Si dès 46 la Numidie avait politiquement disparu, le nom lui survécut, désignant soit parfois la province d'Africa Noua, soit le territoire numide traditionnel, dans des sens divers selon les auteurs qui l'employaient (Desanges 1980a, p. 154-157 et 186-188). Sur le plan administratif il n'y avait désormais en Afrique qu'une seule province, l'Afrique proconsulaire, composée de la réunion des deux Africae, Vetus et Noua, et de l'ancien territoire de P. Sittius communément appelé "Confédération cirtéenne "; toute la zone aurasienne au sens large, jusqu'aux bordures du Sahara, correspondait certainement aux territoires gétules laissés à Juba II, roi de Maurétanie depuis 25 avant J.-C. (Desanges 1964). A la suite de l'assassinat de son successeur Ptolémée en 39 après J.-C., cette région fut annexée et rattachée à l'Africa, les premières traces épigraphiques de son occupation apparaissant à la fin du $\mathrm{I}^{\mathrm{er}}$ siècle ap. J.-C. Le reste du royaume fut divisé en deux provinces, à l'ouest la Maurétanie Tingitane, et à l'est la Maurétanie Césarienne séparée de l'Africa par l'Ampsaga.

Pour comprendre comment un, ou plutôt plusieurs concepts de Numidie réapparurent ensuite, il faut rappeler que, devenue en janvier 27 avant J.-C. province du Peuple Romain et confiée au Sénat, l'Afrique était gouvernée par un proconsul de rang consulaire assisté de trois légats, comme en Asie. Par exception, il avait à sa disposition une armée constituée de la IIIe légion Auguste et de ses auxiliaires. Caligula mit fin à cette anomalie en retirant au proconsul le choix d'un légat, désormais nommé par l'empereur et chargé du commandement de l'armée d'Afrique (l'hypothèse ancienne parfois encore soutenue récemment qui voulait que le proconsul ait gardé trois légats semble très peu vraisemblable comme l'ont montré Chastagnol 1964 et Di Vita-Evrard 1984 et 1985). Dans les décennies qui suivirent, tandis que progressaient la pacification et l'organisation des territoires des marges de la province, la zone militaire se déplaçait vers le sud et l'ouest, sans toutefois que les différents légats aient d'abord eu, semble-til, de compétences géographiques précises. Finalement, au plus tard sous Trajan, furent définis les deux « diocèses " de Carthage et d'Hippo Regius (Hippone/Annaba), confiés chacun à un légat du proconsul ; leur limite reprenait partiellement celle qui séparait jadis l'Africa Vetus du royaume Numide, la Fossa Regia, dont le tracé avait fait l'objet d'un nouveau bornage pendant la censure de Vespasien et de Titus en 73-74 après J.-C. (Di Vita-Evrard 1985 et Lepelley 1986 pour le Bas-Empire). Simultanément dut être définie une région militaire, comparable à un troisième diocèse, confiée au legatus Augusti propraetore legionis III Augustae, titre officiel du légat de la IIIe légion. Celui-ci eut désormais sous son autorité une vaste zone s'étendant du Sahara jusqu'à la mer, associant d'une façon quelque peu artificielle l'ancien état de P. Sittius au nord et la 
région aurasienne au sud. C'est également au début $\mathrm{du} \mathrm{II}^{\mathrm{e}}$ siècle au plus tard que furent définies les trois grandes circonscriptions domaniales de Carthage, Hadrumète et Theueste, confiées chacune à un procurateur équestre. D'une façon qui peut surprendre, leurs territoires ne correspondaient absolument pas aux subdivisions provinciales, puisque celle d'Hadrumète reprenait pour l'essentiel semble-t-il les limites d'un Byzacium élargi, la future Byzacène de Dioclétien (Christol 1999), tandis que celle de Theueste englobait à la fois la région d'Hippo Regius et toute la zone militaire; les limites occidentales de celle de Carthage ne sont pas connues avec certitude, mais il paraît sûr qu'elle s'étendait au-delà de la Fossa Regia puisque les domaines impériaux proches de Thugga en dépendaient.

Comme le montre de façon évidente la titulature de certains légats du $\mathrm{II}^{\mathrm{e}}$ siècle après J.C., qui précisent qu'ils commandent l'exercitus Africae (CIL VIII, 17891 et AE, 1908, 237 vers 110 ; CIL XI, 3718 en 121-123), ou sont légats de la province d'Afrique (ILAlg, II, 1, 623 en 140-141 ou CIL VIII, 2747 et 18273 en 147-149; cf. aussi AE, 1937, $122=1971,507=$ 1973, 629 qui mentionne peut-être la province d'Afrique vers 200), la zone confiée au légat légionnaire continuait à faire partie de l'Africa, et constituait donc bien un troisième diocèse, même si le proconsul d'Afrique n'y intervenait plus, y compris dans le territoire de la Confédération cirtéenne, pourtant plus civil (la dernière mention connue d'un proconsul date de 77-78 après J.-C, ILAlg, II, 1, 551 à Cirta).

Dans les faits, la décision de Caligula, puis les différentes étapes de l'organisation administrative de l'Afrique, des Flaviens au règne de Trajan, provoquèrent donc la création d'une quasi-province dépendant de l'empereur, séparée dans les faits de l'Africa dont elle faisait pourtant toujours formellement partie. Le nom même de Numidie pour la désigner apparaît peut-être au milieu du II ${ }^{\mathrm{e}}$ siècle après J.-C., si Tuscius Fuscianus, Numidiae legatus destinataire d'un rescrit d'Antonin le Pieux, doit bien être identifié à L. Matuccius Fuscinus, légat légionnaire en 158 (Thomasson 1996, p. 151-152, $n^{\circ} 31$ i).

La création d'une province véritablement autonome de Numidie est attribuée à Septime Sévère, à une date qui reste imprécise (Pflaum 1957; Speidel 1973); à partir de 208 apparaît dans les textes épigraphiques la mention d'une prouincia (splendidissima) Numidia, confiée comme auparavant à un sénateur de rang prétorien, un legatus Augusti propraetore, en poste à Lambèse. On notera cependant que sa titulature, dans les années qui suivirent, ne fait que relativement rarement référence à la nouvelle province, y compris pendant la période où la III légion Auguste fut dissoute, entre 238 et 253.

En application de «l'édit de Gallien » vers 261-262, cette zone passa ensuite sous l'autorité d'un chevalier qui portait le titre de uir perfectissimus praeses prouinciae Numidiae, souvent abrégé en VPPPN ; aucune modification territoriale ne semble être intervenue à cette occasion. Cette situation dura sans changement jusqu'au règne de Dioclétien, lorsqu'en 303, dans le cadre des grandes réformes provinciales, l'ancien diocèse légionnaire devenu province fut divisé en deux parties, la Numidia Militiana au sud et la Numidia Cirtensis au nord, chacune toujours placée sous l'autorité d'un praeses perfectissime, dont les capitales étaient respectivement Lambèse et Cirta. Cette partition renouait en un sens avec le passé en recréant l'entité qu'avait été la Confédération cirtéenne, séparée de la zone militarisée correspondant certainement pour l'essentiel aux territoires gétules de Juba II. D'une façon très étonnante cette situation historiquement fondée fut éphémère puisqu'en 314 la province fut réunifiée 
sous l'autorité d'un gouverneur unique, résidant soit à Lambèse, soit déjà à Cirta (Kolbe 1962).

Sous Constantin, entre 316 et 320 , la situation évolua de nouveau: le gouverneur redevint un sénateur portant désormais le titre de consularis prouinciae Numidiae, tandis que la capitale fut peu de temps après transférée à Cirta rebaptisée Constantina, la province elle-même étant parfois qualifiée de Constantina ou Constantiniana à partir du milieu du IV siècle. Cette situation ne semble guère avoir changé jusqu'à la fin de la domination romaine en Afrique (Chastagnol 1966).

Pour autant la province n'était pas la seule à être qualifiée de "Numidie ", y compris dans des textes d'allure très officielle. En effet le second diocèse d'Afrique proconsulaire placé directement sous l'autorité du proconsul, celui d'Hippo Regius, logiquement dénommé dioeceseos ou regio Hipponiensis, est aussi fréquemment appelé Numidia à partir du $\mathrm{III}^{\mathrm{e}}$ siècle, et même à plusieurs reprises au IVe prouincia Numidia (Chastagnol 1964), alors que nous l'avons vu, l'ancienne zone militaire voisine est sans contestation possible devenue au $\mathrm{III}^{\mathrm{e}}$ siècle la province de Numidie. Dans les faits, on considérait donc, dès le III ${ }^{\mathrm{e}}$ siècle au plus tard, qu'il y avait en un sens deux Numidies, la province correspondant à l'ancien ressort territorial du légat de la III légion Auguste, et le diocèse d'Hippone en Afrique proconsulaire ${ }^{1}$. Ce sont ces deux entités qui étaient par ailleurs regroupés dans un service fiscal unique, dont le responsable était un procurateur per... tractum utriusque Numidiae, circonscription parfois appelée simplement elle aussi Numidia (Christol 1994). Cela explique sans nul doute les hésitations, en réalité sans fondements réels, de certains savants des $\mathrm{XIX}^{\mathrm{e}}$ et $\mathrm{XX}^{\mathrm{e}}$ siècles sur l'appartenance d'une ville comme Theueste à la Proconsulaire, sans parler des cartes récentes qui la localisent toujours en Numidie (mise au point de Di Vita-Evrard 1988 ; aussi Chastagnol 1964, p. 11 pour d'autres cités).

L'essor du christianisme et l'organisation de l'église d'Afrique ne firent ensuite que compliquer encore les choses, car apparait au IV e siècle une "province » de Numidie ecclésiastique qui bizarrement ne correspond ni à la province, ni au diocèse de Proconsulaire, ni à la réunion de ces deux ensembles. En effet, ce cadre territorial englobait certes la totalité de la province civile du même nom, mais débordait aussi vers l'est, sans atteindre toutefois la limite des deux diocèses d'Hippone et de Carthage que constituait apparemment toujours la Fossa Regia. Comme on le constate sur une carte (Lancel 1986), les cités d'Hippo Regius, Calama, Thubursicu Numidarum, Madauros et Theueste, pour ne citer que les principales, bien qu'incontestablement situées en Proconsulaire, relevaient de la Numidie ecclésiastique. On considère certes parfois que cet ensemble est un souvenir de l'ancien royaume numide (Lancel 1986), dont les limites, oubliées ou méprisées pendant plusieurs siècles d'administration romaine, auraient ainsi resurgi vers les années 300 après J.-C., en oubliant toutefois que celui-ci s'étendait jusqu'à la Fossa regia, limite officielle de l'Africa Vetus. Il faut donc plutôt se demander si des cadres administratifs romains n'ont pas été repris lorsque fut définie cette Numidie ecclésiastique. L'hypothèse la plus vraisemblable, développée ailleurs, serait d'admettre que ses limites correspondaient approximativement avec celles de la procuratelle domaniale de Theueste telle qu'elle fut définie sous Trajan.

$63 \mathrm{Au}$ début du $\mathrm{V}^{\mathrm{e}}$ siècle, donc à l'époque d'Augustin, Orose (Hist. I, 2, 45 ou 92 selon les éditions) cite la Numidie comme suit : Numidia ubi Hippo Regius et Rusiccada ciuitates sunt. Non seulement il considère encore Hippone comme numide, mais il réunifie en quelque sorte la Numidie en l'associant à Hippo Regius, une cité jadis cirtéenne. 
Pendant toute l'époque romaine le mot Numidie a donc recouvert des réalités extrêmement diverses et assurément complexes (Berthier 1969, et surtout Desanges 1980b, p. 89). D'une manière quelque peu paradoxale, comme le notait J. Desanges (1980b, p. 83), la création de la province au III siècle ne réussit ni à imposer un "concept romain de Numidie, maintenant précis et clair", ni à empêcher son extension à d'autres espaces qui pouvaient évoquer de près ou de loin la Numidie traditionnelle.

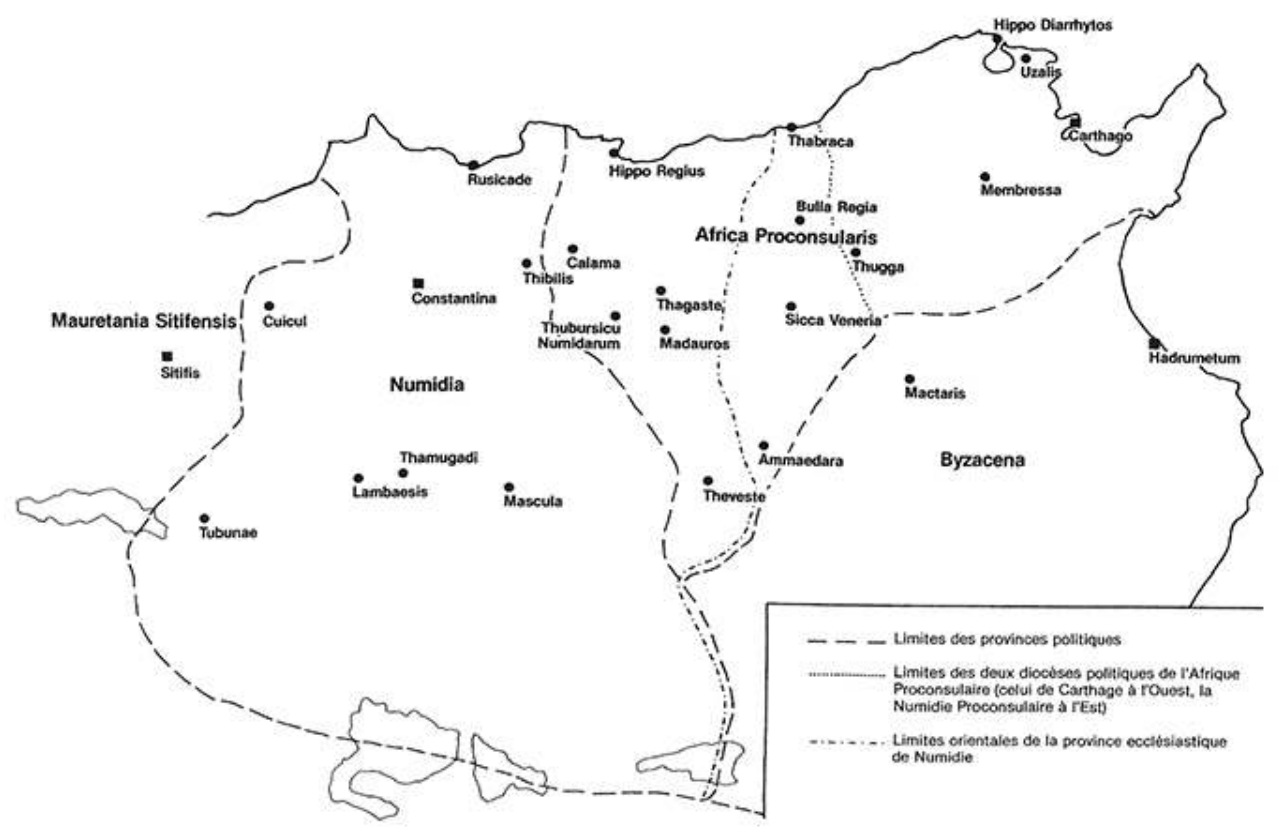

Fig. 3. LIMITES ADMINISTRATIVES ET ECCLÉSIASTIQUES DE LA NUMIDIE AU BAS-EMPIRE. D'APRÈS CL. LEPELLEY 1986, COL. 191-192.

\section{LA NUMIDIE CHRÉTIENNE} Numidie chrétienne se confond presque avec celle de l'Afrique chrétienne, dont des récits complets (des origines à l'Islam) et documentés (avec l'indication des références aux principaux documents) ont déjà été faits et sont disponibles de manière pratique (Audollent 1912 ; Monceaux 1901-1923 ; Histoire du Christianisme, I : Saxer, p. 579-623 ; II : Y. Duval, p.127-133, Ch. Pietri, p. 229-248, 435-451; III : Y. Modéran, p. 247-278 et p.699-717), aussi nous contenterons-nous ici de souligner quelques-unes de ses particularités, notamment son organisation et l'importance des controverses religieuses dans la région. 
par Cyprien entre 248 et 258. Les Sententiae episcoporum de 256 témoignent d'une trentaine d'évêchés numides dont une vingtaine sont identifiables. C'est alors que s'affirma une Église numide groupée autour d'un noyau d'évêques, manifestement conduits à cette époque par celui de Lambèse en raison du rôle politique et militaire de la capitale provinciale (Y. Duval 1984, p. 498 et 1995, p. 77-78 et sq.) même si la population chrétienne ne fut jamais très nombreuse dans cette ville.

71 Les différentes persécutions amenèrent leur lot de martyrs numides, ainsi la Passio Mariani (BHL 131) cite des martyrs de la persécution de Valérien (259) : Agapius, évêque inconnu par ailleurs, Secundinus, évêque identifié à l'homonyme de Cedias sur la liste de 256, Jacques et Marien, clercs de Cirta, Emilien, Tertulla, Antonia.

\section{Liste des primats}

Si la suprématie de l'évêque de Carthage fut toujours reconnue, une organisation plus spécifiquement numide apparut au début du $\mathrm{IV}^{\mathrm{e}}$ siècle avec l'émergence d'une primatie confiée non plus à l'évêque de Lambèse, mais au prélat le plus ancien de la Numidie chrétienne. On connaît quelques-uns de ces primats de Numidie :

\begin{tabular}{|l|l|l|l|}
\hline Primats attestés & Dates & Siège & Localité actuelle \\
\hline Secundus 1 & $302,311-2$ & Tigisi & Aïn el-Bordj \\
\hline Megalius & $395 ;+397$ & Calama & Guelma \\
\hline Crescentianus 3 & 397 & $?$ & \\
\hline Sanctippus & 401,411 & Thagura & Taoura \\
\hline Silvanus 6 & $411,+419$ & Summa & $?$ \\
\hline Valentinus 2 & $402-422 ?$ & Vaiana & $?$ \\
\hline
\end{tabular}




\begin{tabular}{|l|l|l|l|}
\hline Missor & 525 & & \\
\hline Firmus & 551,553 & Tipasa & Tifech \\
\hline Adeodatus & 593,598 & & \\
\hline Victor & 602 & & \\
\hline Colombus & 646 & & \\
\hline
\end{tabular}

Tableau : les Primats de Numidie connus.

LES NUMÉROS RENVOIENT AUX NOTICES de MANDOUZE (PCBE 1982, DE 303 À 533).

Les contours de leur primatie ne coïncidaient pas avec les limites provinciales romaines, vandales, ou byzantines (Modéran 2003a, p. 369). Le mot Numidie demande lui-même à être explicité. Pour Augustin, ce mot désignait parfois seulement l'ensemble des hauts plateaux situés au nord de l'Aurès et des Nemencha (Desanges 1963, p. 50-51; 1995, p. 99), mais pour lui également, la Gétulie était simplement la moitié sud de la province ecclésiastique de Numidie. Le territoire ecclésiastique considéré comme numide s'avançait assez loin au sud et au sud-est des massifs montagneux, dans des régions arides où des Gétules avaient conservé des structures tribales au $\mathrm{IV}^{\mathrm{e}}$ siècle (Modéran 2003a, p. 460-461), mais où existaient aussi des municipes comme Gemellae, Badias, Nigrenses Maiores, ce qui montre l'imbrication des territoires "urbains " et tribaux. Les conciles de 411 et de 484 considèrent par exemple comme numides les évêques de Mesarfelta, Vescera (Biskra), Thabudeos (Thouda), Gemellae, Badias (Badès), Nigrenses Maiores (Négrine) en 411 (Lancel 1991, carte dépliante) et Midila en 484 (Lancel 2002 a et b, carte dépliante p. 413 ; N. Duval et Saxer 1998), ceci sans oublier nombre d'évêchés non localisés.

\section{Les controverses religieuses}

Les questions doctrinales présentèrent dès les années 225 une importance particulière, notamment celle de la validité du baptême conféré par des hérétiques et de la nécessité du re-baptême auquel l'Église d'Afrique fut d'abord attachée. Si la question concernait toute l'Afrique, les controverses religieuses prirent en Numidie une acuité singulière, notamment celle qui portait sur le comportement à tenir vis-à-vis des évêques qui avaient faibli lors des persécutions.

\section{Catholiques et donatistes}

Bien que courte, la persécution de 303 paraît avoir été la plus violente. Elle secoua tout l'Empire. Le procès-verbal de la saisie des biens de l'Église de Cirta (Gesta apud Zenophilum, cf. Y. Duval 1993 et 2000) donne des idées concrètes à la fois sur le poids économique et l'ameublement liturgique d'une communauté chrétienne et sur la forme de la persécution. Nombre de fidèles et d'évêques faiblirent et succombèrent. La paix revenue, se posa la question des lapsi, ceux qui avaient sacrifié aux dieux païens ou qui avaient livré des textes sacrés. Leur exclusion de l'Église fut réclamée notamment par un extrémiste numide, Donat, originaire des Casae Nigrae dont la localisation est longtemps restée incertaine (Ferron 1949). Bien que ce toponyme soit assez banal, on 
peut le placer avec quelque vraisemblance dans les Nemencha, à Henchir Touta (Gsell, Atlas, f. 39, 227, add.) où des tessons le mentionnent. Les partisans de Donat, les Donatistes, se multiplièrent. Le Donatisme, qui allait marquer toute la vie de l'Église d'Afrique jusqu'à sa disparition, ne présentait pas de divergence doctrinale avec le catholicisme au-delà de la validité des actes des lapsi. Les Donatistes se différencièrent cependant très tôt par une sorte d'adoration exclusive et farouche pour les saints et par une vénération passionnée des reliques qui leur attira une moquerie de saint Augustin : "Si d'Orient, on leur apporte des reliques, ils les adorent " (Augustin, Lettre 52, 2; cf. Monceaux, t. V, 1920, p. 37-38).

Le Donatisme prit vite en Numidie un aspect particulièrement violent. Les donatistes y étaient majoritaires en nombre, mais aussi, et pour longtemps, en autorité morale. Ils comptaient dans leurs rangs de fortes personnalités, évêques, mais aussi chefs de guerre (Donat de Bagaï ${ }^{*}$ et Optat de Timgad), de grands controversistes comme Parménien et Pétilien, alors que le parti catholique ne comportait pas de grands hommes (à l'exception d'Optat* de Milev). Seule la personnalité d'Augustin d'Hippone devait inverser les forces intellectuelles au début du V $\mathrm{V}^{\mathrm{e}}$ siècle. Mais il était bien tard. En 411, la Numidie fut représentée à la Conférence de Carthage, par 64 évêques catholiques et 71 donatistes.

77 Les luttes furent particulièrement sévères, avec la prise de basiliques catholiques par des donatistes, des destructions, des tueries, des martyrs aussi des deux côtés, les Donatistes ayant un goût particulier (allant jusqu'au suicide) pour cette forme de mort qui leur assurait le ciel.

\section{Catholiques, donatistes et Vandales}

L'arrivée des Vandales ariens se traduisit par la persécution des catholiques (Modéran 2003 a et b), et dans une moindre mesure des donatistes. Alors qu'elle s'appliquait avec rigueur dans les territoires possédés directement par les Vandales (l'ancienne Proconsulaire, où se trouvaient les Sortes Vandalorum, les " lots des Vandales »), elle fut beaucoup plus modérée dans les autres provinces que le roi s'était réservées. Un extraordinaire document réédité par S. Lancel (2002), la Notitia Provinciarum et civitatum Africae (rédigée d'abord en 484 et révisée quelque temps plus tard) cite encore 125 évêchés numides.

\section{Catholiques et Byzantins}

La coexistence entre les catholiques autochtones et les catholiques byzantins, de langue grecque, ne semble pas avoir posé de problèmes particuliers. L'Église catholique, qui avait applaudi à sa délivrance, avait rapidement obtenu la restitution de basiliques confisquées et avait procédé à la nomination de nouveaux évêques. Les régions numides furent elles-aussi entraînées dans les querelles religieuses byzantines, notamment celle des Trois Chapitres. Firmus, évêque de Tipasa (Tifech), primat de Numidie, dut même se rendre à Constantinople à ce sujet (551). La réintégration des Donatistes toujours présents posait de multiples questions évoquées par le pape Grégoire dans plusieurs lettres de 591 à 596 (cf. Y. Duval 1991). 

grâce à un précieux Inventaire des basiliques chrétiennes d'Algérie (Gui, Duval \& Caillet 1992 ; cf. également Berthier 1943). À côté de monuments urbains très importants et de grande qualité comme la grande basilique de Tébessa (fin du IV ${ }^{e}$ siècle), l'évangélisation des campagnes se traduisit par la multiplication de petites églises modestes, souvent peu décorées, ou de manière rustique, souvent en relief plat, de motifs figurés ou géométriques simples (Baratte 2005), aux dédicaces parfois correctes, parfois truffées de barbarismes, qui témoignent d'une foi simple et profonde, dans laquelle le culte des martyrs prenait une grande part (Y. Duval 1982). Malheureusement ces édifices sont pour la plupart mal datés, et rares sont ceux que l'on peut rapporter à une période déterminée ( $\mathrm{N}$. Duval 2006). Sauf exception, on ne sait d'ailleurs pas quel culte, catholique, donatiste, ou même arien pour quelques-unes, elles abritaient (N. Duval 2006, p. 124-125), tant les différences matérielles entre les cultes étaient faibles et/ou périssables (Michel 2005).

\section{Chrétiens et Musulmans}

La chute de Carthage en 698 n'entraina pas une conversion immédiate à la foi musulmane, cependant l'Église de Numidie disparut lentement dans le silence des sources (cf. ci-dessous, §X).

\section{LA NUMIDIE VANDALE}

83 [Cette partie aurait dû être rédigée par le regretté Yves Moderan. Après son décès, elle a été rédigée au plus près de ses travaux par J.-P. Laporte]

Depuis la synthèse de C. Courtois (1955), les connaissances sur les périodes romaine tardive, vandale et byzantine ont été renouvelées, tant du point de vue religieux que politique et militaire, par les recherches d'Y. Modéran (notamment 1999, 2003a et b, 2010 et 2011) ainsi que par une synthèse de N. Duval (2006) sur les acquis et les questions en suspens.

La conquête foudroyante de l'Afrique du Nord par les Vandales se traduisit de 429 à 439 par la création d'un royaume autour d'Hippo regius, avec Cirta, et Calama, en ne tenant guère compte des anciennes limites des provinces romaines (Desanges 1999, p. 193 ; Modéran 1999, p. 246, carte 2 a et 2003a) : il couvrait en fait peu ou prou la Numidie du nord, à laquelle étaient jointes deux petites parts de la Sitifienne et de la Proconsulaire.

Après la prise de Carthage en 439, le royaume vandale se déplaça en quelque sorte d'un bloc vers l'est. Le traité de 442 rendit notamment à l'Empire non seulement les Maurétanies, mais aussi la Numidie occidentale (avec Cuicul et Milev). Pendant plus de quinze ans, cette dernière parait avoir constitué une province romaine unique avec la Maurétanie Sitifienne (Y. Duval, 1970 ; N. Duval et Chastagnol 1974, p. 112 ; Modéran 1999, p. 251). Après la mort de Valentinien III en 455, Genséric reprit possession de la Numidie occidentale (fig. 4), de la Maurétanie sitifienne et même de quelques villes de la côte maurétanienne (Desanges 1999, p. 193 ; Modéran 1999, p. 246, carte 2 b et 2004).

Encyclopédie berbère, 34 | 2012 
Genséric établit son peuple dans l'ancienne Proconsulaire, en donnant à ses guerriers et à leurs familles des terres héréditaires et non imposables, les sortes Vandalorum (lots des Vandales), tout en se réservant les autres territoires. Les anciennes limites administratives des provinces romaines semblent avoir subsisté, mais avec une valeur limitée dans le contexte du royaume vandale. En conséquence, la notion sous-jacente de Numidie traditionnelle put reprendre un temps de la force (Desanges 1999, p. 193). Le terme de « Maures » continua sa poussée vers l'est, au point de recouvrir à l'époque byzantine toutes les tribus qui refusaient la domination politique de l'occupant (Desanges 1999, p. 196). Ceci concerna au premier chef les populations du sud qui semblent avoir progressivement échappé à la domination vandale après la mort de Genséric (477), dont le nom avait suffi à faire peur. Les rois vandales semblent s'être contentés d'une reconnaissance de leur suzeraineté par les chefs maures qui tenaient toujours à se voir reconnaître leur pouvoir par la remise d'insignes traditionnels (Procope, Guerre vandale, I, 25, 3-8 ; cf. Modéran 2003a, p. 487-488), selon des rituels déjà attestés pendant la période romaine.

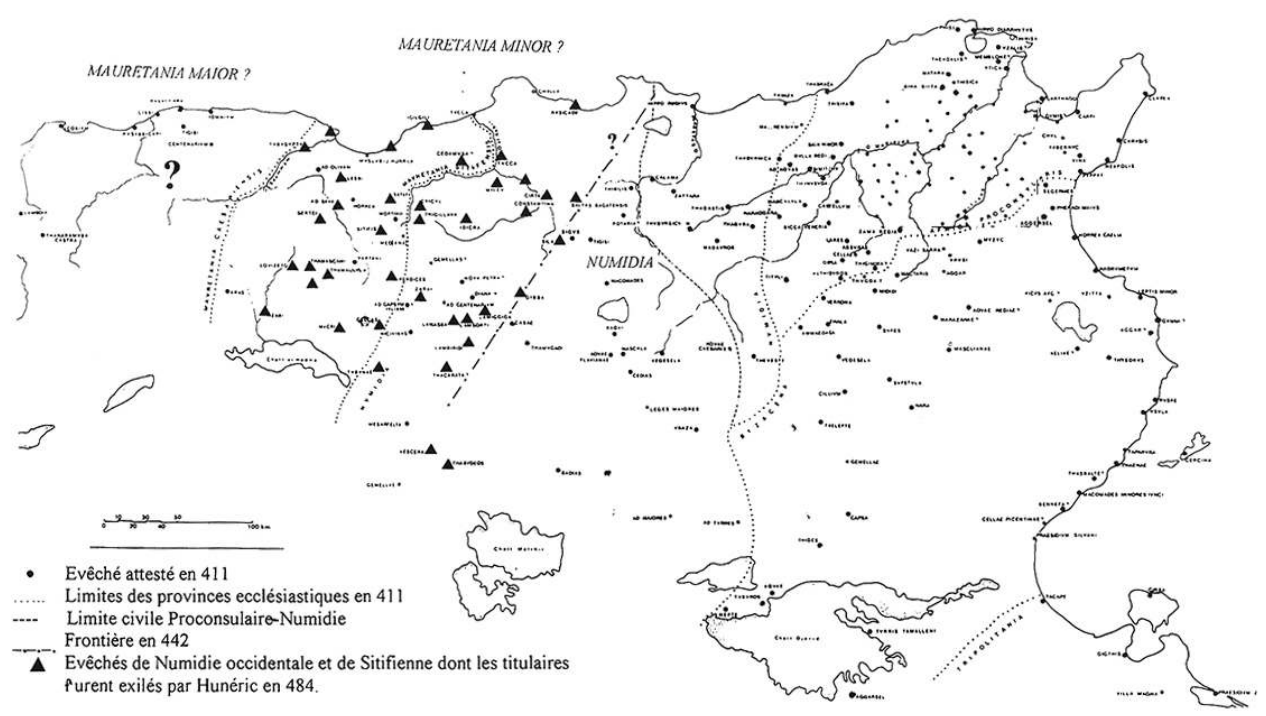

Fig. 4. extension probable du royaume vandale APrès 455. D'APrès modéran 1999, P. 262, CARTE 3.

L'année 484 semble avoir constitué un tournant. Au début de cette année en effet, Hunéric convoqua les évêques catholiques à Carthage et les somma de se convertir à l'arianisme. Il exila ceux qui refusèrent, soit dans diverses îles de Méditerranée, soit en les livrant, ainsi que d'autres catholiques, à des chefs maures restés païens du nord et de l'est du Hodna: à Thubunae (Tobna), Macri (médiévale Maqqara), et "Nippis» (probablement Nicivibus/Nicives, N'Gaous*).

C'est peut-être contre cette persécution que les chefs des Aurès et des Nemencha, apparemment chrétiens, se révoltèrent dans la seconde partie de l'année, ainsi Masties*, dont nous reparlerons plus loin. Dès 483-484, l'Aurès échappait au contrôle des Vandales (Procope, B. V. I, 8,5) et paraît avoir dès lors conservé une réelle autonomie, même si certains actes de propriété dressés dans leur territoire pouvaient encore être datés en citant le nom du roi vandale (ainsi les ostraca de Bir Trouch datés du règne de Gunthamund entre 484 et 493 ) et les tablettes Albertini, enfouies peu après avril 496. 
Même si le règne d'Hildéric (523-530) passa pour une période heureuse, Antalas* et Cusina* vainquirent une armée vandale en 529. En début 533 Hadrumète et Sullecthum durent improviser des remparts de fortune pour pallier la menace des Maures qui semblaient former un bloc compact autour d'Antalas. De son cote, l'aurasien Iaudas* progressait avec ses troupes jusqu à Tigisi (Aïn el-Bordj). Le royaume vandale reculait partout à la veille de l'arrivée des Byzantins.

\section{LA NUMIDIE BYZANTINE} on ignore souvent l'ampleur réelle (la construction d'une forteresse pouvant être présentée comme celle d'une ville entière, peut-être à titre prospectif). Des documents comme la correspondance de Grégoire au début du $\mathrm{VII}^{\mathrm{e}}$ siècle puis le plomb de 
Télergma montrent les institutions romano-byzantines et l'Eglise catholique encore bien en place dans des parties urbanisées du centre et du sud de la Numidie.

Cependant, les grandes affaires furent militaires. Revendiquant leur autonomie, voire leur indépendance, les populations maures du sud (Aurès, Nemencha, etc.) révoltées depuis la fin de l'époque vandale, persistèrent dans la rébellion à l'époque byzantine, sous la conduite de chefs dont $\mathrm{Y}$. Modéran a commencé à éclairer et expliquer les motifs (jadis négligés comme ceux de tout «barbare»), les tactiques et les réalisations (2003a). Ces révoltes n'excluaient pas le contrôle byzantin de certains points d'appui sur les routes du Sud.

Il y avait au moins en théorie une armée byzantine par province. La plus puissante fut celle de Numidie, dont le chef, le dux, résidait en principe dans la ville fortifiée de Tigisi (au VII ${ }^{e}$ siècle d'après une inscription de Timgad). Commandant le secteur le plus étendu, mais aussi le plus menacé, il devint à plusieurs reprises commandant en chef et parfois exarque au VII ${ }^{e}$ siècle. L'un de ces chefs successifs, Pierre, devenu probablement exarque par la suite (d'après le "plomb de Telergma»), portait déjà sur son sceau de dux Numidiae le titre prestigieux de patrice (N. Duval 2006, p. 130 et 131).

Dès les années 539-540, nombre de centres urbains byzantins de Numidie avaient été protégés par des enceintes monumentales (Pringle 1981 et 2001 ; N. Duval, 1983, 2006, p. 138). On construisit d'autres remparts sous Tibère II (578-582), après la révolte de Garmul (Modéran 2003a, p. 81 et 674). Une bonne part de ces murailles fut réutilisée pendant tout le Moyen Age. Plusieurs ont subsisté jusqu'à nos jours (Tigisi, Theveste, etc.). On note également la construction dans la campagne de tours construites de remplois ( $\mathrm{N}$. Duval 2006, p.138). Elles paraissaient naguère isolées, mais les vues satellitaires les montrent parfois entourées d'enceintes plus légères (aujourd'hui peu apparentes au sol) qui pourraient avoir abrité une partie de la population des alentours, comme la basse-cour autour du donjon des châteaux-forts du Moyen-Âge européen (Laporte 2006, p. 137).

Ces fortifications ne suffisaient pas. Sans doute pour ne pas abandonner la protection de sa province menacée au sud par les Maures, le Patrice Pierre, déjà cité, refusa de conduire son armée en Égypte contre les Arabes. Son successeur Grégoire allait succomber sous leurs coups en 647 devant Sufetula (Sbeitla). 


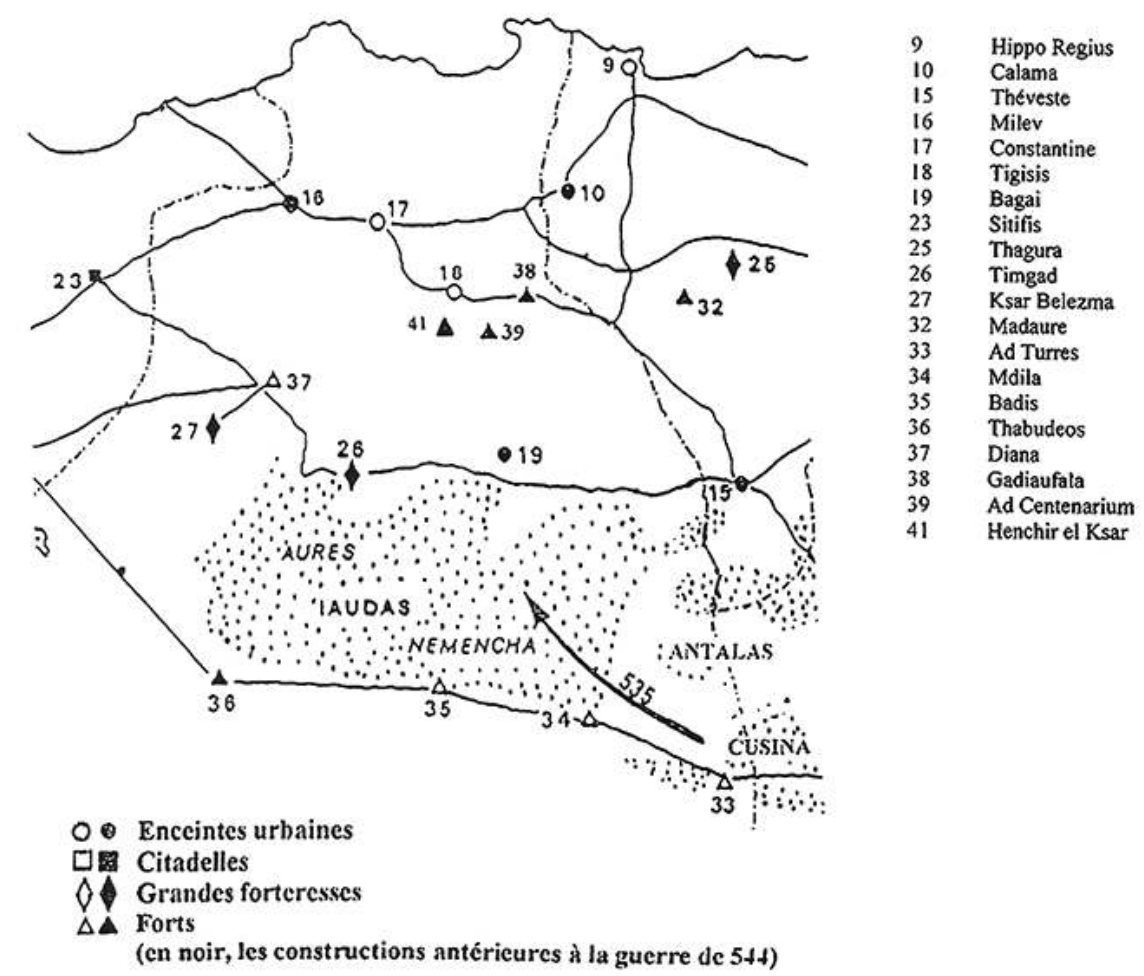

Fig. 5. Les forteresses de JUStinien en NUMidie. CARTE y. MOdÉRAN 2003, P. 600, Complétée PAR X. DUPUIS 2010, P. 508, fig. 2.

\section{CHEFS, ROIS ET ROYAUME(S) BERBÈRES DE LA FIN DE L'ANTIQUITÉ}

101 L'une des constantes de l'histoire de l'Afrique du Nord est de nommer les périodes d'après les maîtres successifs de Carthage, ce qui occulte souvent une partie des réalités plus au sud, notamment dans la partie méridionale de la Numidie. Si elle fut bien «romanisée» sous l'Empire, comme le montre l'exploration archéologique et épigraphique de l'Aurès et des Nemencha, cette région semble avoir eu des liens beaucoup plus lâches avec le pouvoir central aux époques suivantes. Alors réapparut le vieux fond berbère qui avait subsiste a travers et en dépit de la "romanisation » apparente. Les travaux d'Yves Modéran ont récemment éclairé le sort de cette Afrique oubliée, celle des Maures qui habitaient notamment le sud de la Numidie et de la Byzacène.

Sur une large bande allant du Hodna à Gafsa (Capsa), en passant par l'Aurès et les Nemencha sont attestés à plusieurs reprises, sous des appellations diverses, des chefs dont le pouvoir semble résulter d'une évolution de la préfecture de tribu et/ou des reguli (petits rois) du Haut-Empire. Ils étaient alors investis d'un pouvoir local en échange de la reconnaissance de la souveraineté impériale et de la fourniture à la demande de troupes auxiliaires. Pendant le Bas-Empire, leur situation tendit à se rapprocher plutôt de celle des foederati, liés à l'Empire par un traité. Leur nom prenait le pas sur celui des ensembles tribaux qu'ils commandaient.

C'étaient des personnages à l'identité complexe. Ils étaient considérés comme Maures dans la mesure où ils n'étaient pas intégrés au monde des cités et appartenaient à une 
gens autochtone. Mais ils étaient pour la plupart fortement imprégnés de romanité (la mère de Cusina était romaine), habitués du voisinage et des relations avec les cités organisées à la romaine.

Dès l'époque de Genséric (entre 439 et 477), des territoires considérés autrefois comme romains, étaient en fait entre les mains de chefs autochtones comme le roi païen Capsur, dans les monts Caprapicti. Genséric lui avait envoyé, pour les garder en exil, des catholiques qui s'étaient aussitôt mis à évangéliser leur entourage (Victor de Vita, I, 35-37, éd. S. Lancel, 2002a, p.113-114). Capsur lui demanda ce qu'il fallait faire; la réponse $d u$ roi vandale se traduisit par une mise à mort brutale des exilés. Une hypothèse de S. Lancel (ibid., p. 412), qui reste toutefois à argumenter, place le royaume de Capsur entre Tébessa, Négrine, Gafsa et Fériana (Thelepte).

Alors que ces chefs s'étaient globalement accommodés du pouvoir de Genséric, la fin du $\mathrm{V}^{\mathrm{e}}$ siècle vit une nette évolution vers la répudiation du pouvoir vandale, notamment après 484. Masties, apparemment chef dans l'Aurès depuis 427-433, savait le latin et était chrétien. Il se révolta vers 484 , et fut semble-t-il le premier maitre de l'Aurès indépendant. Il se qualifiait lui-même d'imperator et de rex, ce qui, pour J. Desanges (1996, p. 183-188 ; cf. Y. Modéran 2003 a, p. 402) pourrait correspondre à un pouvoir tribal, imperator devant être considéré comme un équivalent d'amenokal*, chef d'une confédération de tribus. Dès lors de larges parties du sud de la Numidie semblent avoir bénéficié d'une sorte d'indépendance relative. Cet ensemble de faits est assez clair. Comme l'a bien noté Y. Modéran ( $E B, X X X, 2010$ p. 4673, à propos de Masties), seuls des préjugés historiographiques ont empêché jusqu'à une période récente de placer le royaume aurasien de la fin $\mathrm{du} \mathrm{V}^{\mathrm{e}}$ siècle dans la même catégorie que les royaumes barbaro-romains des Francs et des Wisigoths en Europe. Cette remarque vaut aussi par la suite.

106 Après une période d'accalmie (ou de carence des sources), le royaume des Aurès devait reprendre sa progression. Dès avant 533, une autorité unique s'y était imposée en la personne du chef maure Iaudas, qui avait également établi son autorité sur des régions jadis vandales situées à l'est du massif. Il avançait vers le nord quand arrivèrent les Byzantins, qui le trouvèrent à Tigisis, à $40 \mathrm{~km}$ seulement au sud-est de Constantine (Procope, B. V. II, 13, 5-18).

107 Du côté des tribus, la conquête byzantine consacra le refus du rétablissement d'un pouvoir désormais conçu comme étranger. Toutefois, devant les assauts de Solomon*, les différents chefs attestés se partagèrent, certains soutenant les Byzantins, d'autres les combattant, avec parfois des changements de camp d'une période à l'autre. La paix, inévitable compte tenu de l'épuisement des belligérants, se traduisait finalement par une sorte de reconnaissance mutuelle ou de modus vivendi dont nous connaissons mal les modalités.

108 En 536-537, Iaudas, maître de l'Aurès, soutint la révolte (byzantine) de Stotzas, mais, vaincu, s'enfuit en Maurétanie en 539, avant de revenir et d'accepter une certain tutelle byzantine en 546 .

109 Pour sa part, Cusina, révolté contre les Byzantins en 534 et 535, semble en avoir reçu plus tard une certaine reconnaissance officielle. En 544, il combattit aux côtés des forces impériales contre Antalas et les Laguatan* et aida Jean Troglita*. Ce révolté devenu allié fidèle finit par être assassiné en 563 sur l'ordre de Jean Rogathinos*. 
110 Antalas, qui contrôlait une partie du sud de la Byzacène, révolté en début 544, réunit des troupes venues des Aurès et du Hodna et s'allia avec les Laguatan de Tripolitaine. Battu par Solomon, il le vainquit et le tua à Cillium (Kasserine) en juin 544. L'année suivante, il prit Hadrumète par ruse mais épargna ses habitants (Procope, B. V, II, 23, 26). Manifestement il était là en conquérant et non en simple pilleur. Le petit détachement qu'il avait laisse dans la ville en fut rapidement chassé, également par ruse, mais dès 546 les Maures arrivaient aux portes de Carthage. Seule la venue de Jean Troglita au cours de l'été 546 redressa la situation des Byzantins. Il finit par écraser les rebelles en 548 à la tête de troupes byzantines épaulées par un grand nombre de Maures ralliés. Antalas finit par se soumettre (l'acceptation de sa soumission montre $a$ contrario que les Byzantins avaient besoin de lui d'une manière ou d'une autre).

111 Vers 569-571, une grande insurrection menée par Garmul, agita, non pas la Maurétanie comme on l'a longtemps cru, mais des parties de la Numidie, de la Byzacène, et même de la Proconsulaire (Modéran 2003a, p. 673).

112 Ces affrontements ont probablement duré. Si, faute de sources, nous ignorons ce qui se passa pendant une bonne partie du $\mathrm{VII}^{\mathrm{e}}$ siècle, Koceila* apparaît dans les années 670 comme une sorte d'exarque ou de préfet des Maures à la fois indépendant de fait et reconnaissant une suzeraineté théorique de Byzance. En retour, il avait aussi autorité sur des troupes byzantines en 683 .

113 On peut voir là en gestation un grand royaume assumant à la fois sa berbérité et sa romanité, du même type que celui de Masuna* (qui dès 508 se disait rex Maurorum et Romanorum) et celui des Djedars* dans l'ouest algérien. Sa constitution fut arrêtée par une nouvelle intervention extérieure.

\section{LA NUMIDIE ET LA CONQUÊTE MUSULMANE}

114 La Numidie fut touchée pour la première fois en profondeur par l'expédition d'Okba ben Nafi* (683) qui y affronta à la fois des forces byzantines et maures (détails importants sur les garnisons byzantines et sur l'expédition d'Okba dans Y. Duval 1995). La région fut conquise en 686-688 avec la mort de Koceila. Après l'épisode bref (698-703) mais violent de la Kahena* organisant la résistance, les anciens territoires numides tombèrent assez rapidement entre les mains des nouveaux conquérants, qui les organisèrent à leur manière, peu différenciée à l'origine de celle de l'époque byzantine. Le pays de Zabè byzantin devint le Bilad Zâb.

115 La notion de Numidie semble s'être évanouie à l'époque musulmane, où elle n'apparaît plus que chez des géographes arabes qui témoignent en fait, de manière savante, d'une tradition géographique dérivée du géographe alexandrin Ptolémée (qui décrivait l'Afrique du Nord vers 110 après J.-C.).

116 Sur le plan religieux, l'évolution de la région fut plus lente. Représentant un élément de population largement minoritaire en nombre, l'autorité musulmane dut sans doute composer dans une certaine mesure avec la majorité de la population. Légalement, les chrétiens pouvaient continuer à pratiquer leur religion en s'acquittant d'un impôt spécial, et il n'était pas dans l'intérêt des califes de diminuer trop rapidement la masse imposable. C'est ainsi qu'en Tripolitaine, des cimetières chrétiens attestent de la survivance très tardive de chrétientés locales. 
Longtemps encore, non seulement dans des contrées reculées, mais encore dans les villes, des chrétiens autochtones cohabitèrent avec la population musulmane. Même dans la ville sainte musulmane de Kairouan, il en subsistait encore au $\mathrm{XI}^{\mathrm{e}}$ siècle comme le montrent plusieurs épitaphes latines qui y ont été découvertes. Cependant, le mouvement était inexorable. La plupart des dernières communautés chrétiennes urbaines autochtones semblent avoir disparu avec la conquête almohade au XII ${ }^{\mathrm{e}}$ siècle, parfois par l'exil (c'est le cas d'une église de Mahdia dont l'équipement liturgique fut transporté à Palerme), souvent sans doute par conversion sur place.

C'est à l'archéologie qu'il faudra à l'avenir demander ce que les textes ne peuvent dire. Elle devrait permettre un jour de constater la désaffection d'églises rurales et/ou leur transformation en mosquée. Les connaissances archéologiques, jadis insuffisantes pour en déceler les traces (pour autant que l'on se soit posé la question), devraient aujourd'hui permettre de relever (voire de dater) des indices ténus mais sans doute présents. Il y a là un enjeu majeur de l'archéologie médiévale de l'Afrique du Nord. Les antiques églises chrétiennes de Numidie, dont beaucoup n'ont pas été fouillées, pourraient fournir sur ce point un large champ d'étude.

\section{BIBLIOGRAPHIE}

ALEXANDROPOULOS J.,

2000 - Les monnaies de l'Afrique antique 400 av. J.-C.-40 ap. J.-C., Toulouse, 505 p. (et nouvelle édition 2007).

2002 - « L'usage du grec en Afrique du Nord, d'après les documents monétaires (1er s. av. J.-C. $\mathrm{I}^{\mathrm{er}}$ s. ap. J.-C.) », AntAfr. 38-39, p. 289-296.

AUDollent A., 1912 - « Histoire de l'Afrique chrétienne », in Dictionnaire d'Histoire et de Géographie Ecclésiastique (DHGE), I, col. 706 à 862.

BARATTE F., 2005 - « La sculpture en Numidie à la fin de l'antiquité », Saint Augustin. La Numidie et la société de son temps, Bordeaux, p. 153-171.

BENABBÈs M., 2011 - « Des provinces byzantines à l'Ifriqiya - continuités et changements dans les découpages administratifs ", dans Provinces et identités provinciales dans l'Afrique romaine, Caen, Publications du CRAHM, p. 271-293.

BÉNABOU M., 1976 - La résistance africaine à la romanisation, Paris, Maspéro, 1976, $2^{\text {e }}$ édition : La Découverte, 2005.

BERTHIER A.,

1943 - avec H. LOGEART et M. MARTIN, Les vestiges du Christianisme antique dans la Numidie centrale, Alger, Direction des Antiquités de l'Algérie.

1969 - « Du mot Numidia accolé aux noms antiques de Constantine », dans AntAfr. 3, p. 55-67.

1981 -La Numidie, Paris 
BOUCHENAKI M., 1979 - « Contribution à la connaissance de la Numidie avant la conquête romaine ", in Die Numider, Cologne-Bonn, p. 75-87. Paru sous le même titre in - Atti del I Congresso Internazionale di Studi Fenici e Punici, P. Bartoloni éd., = Coll. di Studi Fenici, t. 2, 1983, 527-541.

CAILLET J.-P., 2005 - « La réalité de l'implantation monumentale chrétienne au temps d'Augustin l'exemple de quelques cités de Numidie ", Saint Augustin - la Numidie et la société de son temps, Bordeaux, p. 55-66.

CAMPS G., 1960 - Massinissa ou les débuts de l'Histoire (= Libyca, archéologie - épigraphie, VIII), Alger, Imprimerie officielle, $320 \mathrm{p}$.

1973 - « Nouvelles observations sur l'architecture et l'âge du Medracen, mausolée royal de Numidie », CRAI, p. 470-517.

1979 - « Les Numides et la civilisation punique », AntAfr. 14, 43-53.

1984a - «Une monnaie de Capussa, roi des Numides massyles », BCTH (n.s.) - B - Afrique, 15-16, 1979-1980 [1984], p. 29-32.

$1984 \mathrm{~b}$ - « Les derniers rois numides : Massinissa II et Arabion », BCTH (n.s.) - B - Afrique, 17, 1981

[1984], p. 303-311.

1993 - « À la recherche des Misiciri - cartographie et inscriptions libyques », in À la croisée des études libyco-berbères : mélanges offerts à Paulette Galand-Pernet et Lionel Galand, Drouin J., Roth A. (dir.), Paris, p. 113-126.

1994 - « Punica lingua et épigraphie libyque dans la Numidie d'Hippone », BCTH (n.s.) B - Afrique, 23, p. 33-49.

1995 - « Modèle hellénistique ou modèle punique ? Les destinées culturelles de la Numidie », in Actes du $3^{\text {ème }}$ Congrès international des Etudes phéniciennes et puniques, Tunis 1991. Volume 1, Fantar M., Ghaki M. (dir.), Tunis, p. 235-248.

1999 - «Essai de cartographie culturelle : à propos de la frontière de Numidie et de Maurétanie », Frontières et limites géographiques de l'Afrique du Nord antique : hommage à Pierre Salama, Lepelley C., Dupuis X. (dir.), Paris, p. 43-70 (Publications de la Sorbonne, Histoire ancienne et médiévale, 56). 2000 - « Contribution de la cartographie à l'étude des phénomènes culturels berbères ", in Hommes et terres d'Islam : mélanges offerts à Xavier de Planhol, Balland D. (dir.), Téhéran-Louvain, Institut français de recherche en Iran, p. 377-390.

2010 - s. v. Medracen, EB, XXXI, p. 4834-4852.

CHAKER S., 1986 - «A propos de la terminologie libyque des titres et des fonctions », Annali Istituto universitario orientale, 46, p. 541-562.

CHASTAGNOL A.,

1958 - « Les légats du proconsul d'Afrique au Bas-Empire », dans Libyca, 6, p. 7-19, repris dans Id., L'Italie et l'Afrique au Bas-Empire. Scripta varia, Lille, 1987, p. 67-82

1966 - « Les consulaires de Numidie », in Mélanges Jérôme Carcopino, Paris, p. 215-228, repris dans Id., L'Italie et l'Afrique au Bas-Empire. Scripta varia, Lille, 1987, p. 149-162.

CHRISTOL M., 1994 - « Le blé africain et Rome. Remarques sur quelques documents », dans Le ravitaillement en blé de Rome et des centres urbains des débuts de la République jusqu'au Haut-Empire (coll. de l'EFR, 196), Naples-Rome, p. 295-304.

1999 - « Les subdivisions de l'administration domaniale et financière en Afrique romaine : des limites de la procuratèle d'Hadrumète à celles de la province de Byzacène », C. Lepelley et X.

Dupuis (éd.) : Frontières et limites géographiques de l'Afrique du Nord antique. Hommage à Pierre Salama, Paris, p. 71-87. 
COLTELLONI-TRANNOY M., 2003 - «Juba », EB, XXV, p. 3914-3938 (avec un tableau généalogique des rois maures et numides, p. 3914).

CourtoIs C., 1955 - Les Vandales et l'Afrique, Paris, 455 p., XII pl.

DESANGES J.,

1962 - Catalogue des tribus africaines de l'Antiquité classique à l'ouest du Nil, Université de Dakar, Faculté des Lettres et Sciences Humaines, Publications de la Section d'Histoire, $\mathrm{n}^{\circ} 4$.

1964 - « Les territoires gétules de Juba II », REA, 66, 1-2, p. 33-47.

1974 - «Utica, Tucca et la Cirta de Salluste », in Mélanges R. Dion, Littérature gréco-romaine et géographie historique, Paris, p. 143-150. Réimpression dans Desanges, 1999, p. 103-110. 1980a - Édition commentée de Pline l'Ancien, Histoire Naturelle, V, $1^{\text {re }}$ partie, CUF, Paris. $1980 \mathrm{~b}$ - «Permanence d'une structure indigène en marge de l'administration romaine : la Numidie traditionnelle », AntAfr. 15, 1980 (= Hommages à J. Lassus, II), p. 77-89. Article republié dans J. Desanges 1999, p. 181-196.

1982 - Compte rendu de la Numidie d'André Berthier, Revue des Études Latines, 60, p. 469-471. 1987 - « La Cirta de Salluste et celle de Fronton », L'Africa romana, IV/I, 1986 (1987), p. 133-135. Réimpression dans Desanges 1999, p. 111-114.

1989 - « L'hellénisme dans le royaume protégé de Maurétanie (25 av. J.-C.) », BCTH, 20-21, B, 1984-1985 [1989], Afrique du Nord, p. 53-61.

1993 - « L'Afrique romaine et libyco-berbère », in C. Nicolet (éd., Rome et la conquête du monde méditerranéen. Tome 2. Genèse d'un empire, $4^{\mathrm{e}}$ éd., Paris, p. 627-656.

1995 - « Massinissa et Carthage entre la deuxième et la troisième guerre punique : un problème de chronologie ", in Actes du II congrès international des Etudes phéniciennes et puniques (Tunis, 11-16 novembre 1991), I, Tunis, p. 352-358. Reproduit dans Desanges, 1999, p. 71-78.

1999 - Toujours Afrique apporte fait nouveau. Scripta minora, Paris, De Boccard, 406 p. Reproduction de nombreux articles de l'auteur, dont, p. 181-196 : «Permanence », 1980b souvent cité ici. 2007 - « Aspects de l'hellénisme dans l'Afrique du Nord antique », dans La Méditerranée d'une rive à l'autre : culture classique et cultures périphériques, Cahiers de la villa « Kérylos ", 18, Paris, p. 167-184.

à paraître - «L'Aurès des Maurousii et la reconstitution par A. Berthier et son école du Bellum Iugurthinum de Salluste », Aouras, 7.

DIEHL Ch., 1896 - L'Afrique byzantine, Paris.

DIE NUMIDER. Reiter und Könige nordlich des Sahara, Cologne-Bonn, 1979, 674 p. Catalogue

d'exposition, H. G. HORN, Ch. B. RÜGER (éd.).

DI VITA-EVRARD G.,

1984 - « L. Volusius Bassus Cerealis, légat du proconsul d'Afrique T. Clandius Aurelius Aristobulus, et la création de la province de Tripolitaine ", dans L'Africa romana, 2, [1985], p. 149-177.

1985 - « La Fossa Regia et les diocèses d'Afrique proconsulaire », dans L'Africa romana, 3, 1985

[1986], p. 31-58.

1988 - « Une inscription errante et l'« extra-territorialité » de Theveste au IV ${ }^{\mathrm{e}}$ siècle », dans L'Africa romana, 6, 1988 [1989], p. 293-320.

DUPUIS X., 2010 - « Henchir el Ksar et Mila : deux « nouvelles » dédicaces de fortifications byzantines en Afrique », CRAI, p. 505-512 avec carte p. 508, fig. 2.

à paraître - « D'un espace administratif à l'autre : aux origines de la Numidie ecclésiastique », à paraître, Actes du Colloque de Caen, 2009. 
DUVAL N.,

1974 - avec A. Chastagnol, « Les survivances du culte monarchique à l'époque vandale », Mélanges William Seston, p. 87-119.

1983 - « L'état actuel des recherches sur les fortifications de Justinien en Afrique », XXX Corso di cultura sull'arte ravennate e bizantina, t. 30, 1, p. 149-204

1998 - avec V. Saxer, « Un nouveau reliquaire africain et l'évêché Midilensis », Syria, t. 75

(Mélanges E. Will), p. 245-262.

2006 - «L'Afrique dans l'Antiquité tardive et la période byzantine », An Tard. 14, p. 119-164.

2008 - « Numidien, Mauretanien und Africa fzdvonsularis », Reallexikon zur byzantinischen Kunst, fasc. 49 , col. 1-162.

DUVAL Y.,

1970 - « La Maurétanie sitifienne à l'époque byzantine », Latomus, t. 29, p. 157-161.

1982 - Loca sanctorum Africae. Le culte des martyrs en Afrique du IV e au VII siècle, Rome, (collection de l'École française de Rome, t. 58), 2 vol.

1984 - « Densité et répartition des évêchés dans les provinces africaines au temps de saint Cyprien », MEFRA, 96, 1, p. 493-521. Repris et mis à jour dans Y. Duval 2005.

1988 - Auprès des Saints corps et âmes. L'inhumation « ad sanctos » dans la chrétienté d'Orient et d'Occident du III ${ }^{\mathrm{e}}$ au VII ${ }^{\mathrm{e}}$ siècle, Paris, Institut d'Etudes Augustiniennes, $230 \mathrm{p}$.

1991 - « Grégoire et l'Eglise d'Afrique. Les hommes du pape », colloque Gregorio Magno e il suo tempo. Studia Ephemeridis Augustinianum', 33, p. 129-158.

1993 - « Les Gesta apud Zenophilum et la Paix de Maxence, An Tard, 3, p. 55-63.

1995 - Lambèse chrétienne. La gloire et l'oubli. De la Numidie romaine à l'Ifrikiya, Institut d'Études augustiniennes, Paris, $215 \mathrm{p}$.

1995 - Contribution à l'Histoire du Christianisme, t. II.

1998 - « Le gouverneur de Numidie en sa capitale. Le lieu et les acteurs du procès de l'évêque de Cirta en 320 ", An Tard. 6, p. 193-207.

2000 - Chrétiens d'Afrique à l'aube de la Paix constantinienne. Les premiers échos de la grande persécution, Paris, Institut d'Etudes Augustiniennes, 524 pages, 11 figures.

2005 - Les chrétientés d'Occident et leur évêque au III siècle. Plebs in ecclesia constituta (Cyprien, Ep. 63), Paris, Institut d'Études Augustiniennes, 347 p.

FERRON Père J.,

1949 - «Casae Nigrae », Dictionnaire d’Histoire et de Géographie Ecclésiastique (DHGE), XI, col.

$1249-1251$.

1960 - « Donat de Carthage », DHGE, XIV, col. 649-650.

GHAKI M.,

1983 - «Quel sens faudrait-il donner aux termes - libyen, libyphénicien, numide et libyque ?», Turat, I, p. 76-82.

1992-93 - « La répartition des inscriptions libyques », Reppal VII-VIII, p. 93-108. 1993 -

« L'organisation politique et administrative des Numides », À la croisée des études libyco-berbères, Mélanges offerts à Paulette Galand-Pernet et Lionel Galand, Paris, 89-101.

1999 - Les haouanet de Sidi M'hamed Latrech, Tunis, préface de M.H. Fantar, avec deux cahiers photos couleurs hors folio.

2005 - «Quels sens faudrait-il donner aux termes autochtones, libyen, libyque, libyphénicien, numide et maure ? » Studi berberi e mediterranei. Miscellanea offerta in onore di Luigi Serra, a cura di Anna Maria di Tolla, Studi Magrebini n.s. 3, 35-42. [Reprise complétée et modifiée de Ghaki, 1983] 2007 - « Les royaumes numides » Contribution à l'ouvrage : Khélifa Chater (édit.) «La Tunisie à travers l'histoire ». Publications du CERES Tunis, volume I, 95-117. (en arabe). 
sous presse - «Les rapports entre les cités et le pouvoir numide », dans Dix ans depuis le décès de Giovanni Tore (a cura di Carla Del Vais), Université de Cagliari/Sardaigne.

GUI I., CAILLET J.-P, DUVAL N., 1992 - Basiliques chrétiennes d'Afrique du Nord, volume I, Inventaire de l'Algérie, Collection des Études Augustiniennes nº 129-130, Paris, 2 fascicules.

HISTOIRE DU CHRISTIANISME, t. I, II et III, 1995-2000. Articles sur l'Afrique chrétienne de V. Saxer, Y. Duval, Ch. Pietri, Y. Modéran.

JANON M., 2005 - « Numidie », in Dictionnaire de l'Antiquité, J. Leclant (dir.), Paris, p. 1525-1526.

HUSS W., 2006 - « Numidae, Numidia », in Neue Pauly, IX, Leyde-Boston, col. 905-906.

KHERBOUCHE F. \& LAPORTE J.-P., 2010 - « Mausolées monumentaux d'Afrique du Nord », Encyclopédie berbère, fasc. XXX, p. 4578-4778.

KоLBE H.-G., 1962 - Die Statthalter Numidiens von Gallien bis Konstantin (268-320), (Vestigia 4), Munich.

котULA T., 1990 - « Saint Cyprien et les barbares africains (Epist., 62) », in Cristianismo y aculturatión en tiempos del Imperio romano = Antigüedady Cristianismo, VII, Murcie, p. 137-142.

KRANDEL-BEN YOUNES A., 2002- La présence punique en pays numide, Tunis, INP, 487 p.

LANCEL S.,

1971, 1972, 1975, 1991 - Actes de la Conférence de Carthage en 411, t. I, Sources Chrétiennes, vol. 194, Paris, 1972, 402 p. ; t. II (S. C, vol. 195), 1972, 507 p. ; t. III (S. C, vol. 224), 1975, 320 p. ; t. IV (S. C, vol. 373), 1991, $384 \mathrm{p}$.

1986 - « Africa. B. Organisation ecclésiastique », dans Augustinus Lexikon, Bâle-Stuttgart, 1, col. 205-219.

2002a - Édition de Victor de Vita, Histoire de la persécution vandale en Afrique (Collection des Universités de France, Paris, Les Belles Lettres), Paris, 2002.

2002 b - Édition de la Notitia Provinciarum et civitatum Africae, (à la suite de l'édition de Victor de Vita), Belles Lettres, p. 252-272 et introduction p. 223-248.

2005 - Sous la direction de S. LANCEL : Saint Augustin : La Numidie et la société de son temps, Bordeaux (Ausonius, Scripta Antiqua 14).

LAPORTE J.-P., 2003a - «Ptolémée et la Maurétanie césarienne », CRAI, p. 171-195.

$2003 \mathrm{~b}$ - «Zabi, Friki : notes sur la Maurétanie et la Numidie de Justinien », (Colloque L'Afrique vandale et Byzantine, Tunis, octobre 2000) = AnTard, t. 11, p. 151-167.

2006 - « Sousse : La domus de Sorothus et ses mosaïques », CRAI, 2006 (2008), p. 1327-1392.

2008 - « Une contribution méconnue du monde amazigh à l'architecture mondiale : Les grands mausolées d'Afrique du Nord », Actes du colloque L'apport des Amazighs à la civilisation universelle, Alger (2009), p. 137-154.

2010 - «Puniques et autochtones en Algérie », dans Carthage et les autochtones de son empire du temps de Zama (colloque international organisé à Siliana et Tunis du 10 au 13 mars 2004 par l'Institut National du Patrimoine et l'Association de Sauvegarde du site de Zama) = Hommage à Mhamed Hassine FANTAR, coordination Ahmed Ferjaoui, Tunis, p. 379-393.

LAPORTE J.-P. \& A. M'CHAREK, 2010 - « Musulames », EB, XXXII, p. 5144-5154.

LE GLAY M., 1991 - « L'administration centrale de la province de Numidie de Septime Sévère à

Gallien », AntAfr., 27, 83-92

LEPELLEY Cl.,

1979-1981 - Les cités de l'Afrique romaine au Bas Empire, 2 vol., Paris. 
1986 - « Africa. A. Présentation générale », dans Augustinus Lexikon, Bâle-Stuttgart, 1, col. 179-206. 2005 - « Témoignages de saint Augustin sur l'ampleur et les limites de l'usage de la langue punique dans l'Afrique de son temps ", dans Identités et cultures dans l'Algérie antique, C. BriandPonsart éd., Rouen, p. 117-141.

LIPINSKI E. (dir.) - 1992 - Dictionnaire de la civilisation phénicienne et punique, Brepols, 501 p. ; cf. « Numidie », p. 317-319 et notices sur diverses villes de l'Afrique du Nord.

LUISI A., 1979 - « Nomades e Numidia », Contributi dell'Istituto di Storia antica dell'Università del Sacro Cuore, 6, p. 57-64.

MAÏER J.-L., 1973 - L'épiscopat de l'Afrique romaine, vandale et byzantine, Institut Suisse de Rome.

MANDOUZE A. et alii, 1982 - Prosopographie chrétienne du Bas-Empire, I, Afrique (303-533), 1322 p.

M'CHAREK A.,

1999 - « De saint Augustin à Ai-Bakri. Sur la localisation de l'ager Bullensis dans l'Africa latinochrétienne et de Fahs Boll en Ifriqiya arabo-musulmane », CRAI, p. 115-140.

1999 - « De Zama à Kairouan : Thusca et Gamonia », Frontières et limites géographiques de l'Afrique du Nord antique. Hommages à Pierre Salama. Études réunies par Claude Lepelley et Xavier Dupuis, p. 139-182. Discussion, p. 183.

2001 - «Bulla Mensa : une forteresse-refuge de l'Antiquité aux temps modernes », Pallas, 56, p. 83-92.

M'CHAREK F., à paraître - « Le pays des Gnaziz, antique Pagus Gunzuzi », Colloque de Caen, 2009.

MiChel A., 2005 - « Aspects du culte dans les églises de Numidie au temps d'Augustin : un état de la question ", in Saint Augustin : la Numidie et la société de son temps, Bordeaux, p. 67-108.

MODERAN Y., 1999 - « Les frontières mouvantes du royaume vandale », Frontières et limites, Hommages à Pierre Salama, p. 241-263.

2003a - Les Maures et l'Afrique romaine, IV ${ }^{e}-V I I^{e}$ siècles, Bibliothèque des Ecoles Françaises d'Athènes et de Rome, (BEFAR, 314) Rome, 900 p.

$2003 b$ - « Une guerre de religion : Les deux Églises d'Afrique à l'époque vandale », An Tard, 11, p. 21-44.

2010 - « Maurétanies (provinces) », EB, XXXI, p. 4737-4740.

2011 - « Les provinces d'Afrique à l'époque vandale », dans Provinces et identités provinciales dans l'Afrique romaine, Publications du CRAHM, Caen, p. 241-270.

MONCEAUX P., - Histoire littéraire de l'Afrique chrétienne depuis les origines jusqu'à l'invasion arabe, du t. I (1901) au t. VII (1923)

MONCHICOURT Ch., 1913 - La région du Haut Tell en Tunisie (Le Kef, Teboursouk, Mactar, Thala), Essai de Monographie géographique, Paris, 487 p, 17 fig. et cartes.

PFLAUM H.-G., 1957 - « À propos de la date de création de la province de Numidie », Libyca, 5, p. 61-75.

PRINGLE D., 1988 - The defence of byzantine Africa, BAR, 99 ( $2^{\mathrm{e}}$ édition 2001).

RACHET M., 1970 - Rome et les Berbères. Un problème militaire d'Auguste à Dioclétien (=Coll. Latomus 110), $314 \mathrm{p}$

SAUMAGNE Ch., 1966 - La Numidie et Rome, 267 p.

SHAW B. D., 1983 - "Soldiers and Society : the Army in Numidia", Opus 2, p. 133-152 
SPEIDEL M., 1973- « The singulares of Africa and the establishment of Numidia as a province », dans Historia, 22, 1, p. 125-127.

THOMASSON B. E., 1996 - Fasti africani. Senatorische und ritterliche Amtsträger in den römischen Provinzen Nordafrikas von Augustus bis Diokletian, Stockholm.

TROUSSET P., 2003 - «Les limites de la réoccupation byzantine en Afrique », dans L'Afrique vandale et byzantine, Colloque Tunis INP, 5-8 octobre $2000=$ An Tard, t. 11, p. 143-150, fig.

\section{NOTES}

1. L'appellation de « Numidie proconsulaire », ou parfois de " Numidie d'Hippone », désigne de manière pratique la partie de la Numidie traditionnelle comprise dans le ressort de la Proconsulaire. Il s'agit en fait d'une appellation moderne, qui n'a été restituée que de manière très discutable (A. Chastagnol, « Légats du proconsul d'Afrique au Bas-Empire », Libyca a/é, t. 6, 1958, p. 19 = Scripta varia, 1987, p. 79) sur une inscription de Calama (CIL, VIII, 17.518) dont les fragments épars avaient été réorganisés par Gsell, ILAlg, I, 251 et 259, donnant un texte que l'on peut en fait restituer de multiples façons.

\section{INDEX}

Mots-clés : Antiquité, Religion, Protohistoire, Tribu 